\title{
Globally stable control of a dynamic bipedal walker using adaptive frequency oscillators
}

\author{
Gabriel Aguirre-Ollinger*
}

\begin{abstract}
We present a control method for a simple limit-cycle bipedal walker that uses adaptive frequency oscillators (AFO's) to generate stable gaits. Existence of stable limit cycles is demonstrated with an inverted-pendulum model. This model predicts a proportional relationship between hip torque amplitude and stride frequency. The closed-loop walking control incorporates adaptive Fourier analysis to generate a uniform oscillator phase. Gait solutions (fixed points) are predicted via linearization of the walker model, and employed as initial conditions to generate exact solutions via simulation. Global stability is determined via a recursive algorithm that generates the approximate basin of attraction of a fixed point. We also present an initial study on the implementation of AFO-based control on a bipedal walker with realistic mass distribution and articulated knee joints.
\end{abstract}

\section{Introduction}

There is a growing body of research on the control of rhythmic movements in robots by means of coupled nonlinear oscillators. Oscillator-based robot control is inspired in part by biological neural circuits called central pattern generators (CPG's), which control rhythmic movements in vertebrates. CPG-inspired control architectures have been employed, for instance, to generate different gait modalities in artificial bipeds and quadrupeds [1]. Reinforcement learning based on CPG's has been employed to enable automatic control acquisition by a biped robot [2]. In the CPG walker control proposed by Verdaasdonk [3], energy efficiency is accomplished by enabling the oscillator to tune into the resonance frequency of the limbs. However, the CPG's tuning ability requires the intrinsic oscillator frequency to be relatively close to the resonant frequency of the limb. This limitation can be overcome by using nonlinear oscillators with frequency adaptation capabilities. Nakanishi [4] proposed a frequency adaptation algorithm for bipedal walking based on phase resetting. The stabilizing properties of phase resetting in a biped have been investigated by $\mathrm{Fu}$ [5]. Coupled nonlinear oscillator systems are also capable of achieving inter-leg coordination in bipedal walkers as well as coordination among the leg's own segments $[4,6]$.

This paper focuses on adaptive frequency oscillators (AFO's) and their potential use for the stabilizing control of a biped robot. An AFO is a nonlinear oscillator that features a learning component to adapt its intrinsic frequency to the frequency of a periodic or quasi-periodic input signal [7]. Control algorithms based on AFO's allow automated, on-line learning and encoding of dynamical movement primitives by a robot $[8,4,9]$. The encoding of rhythmic movements via dynamical systems not only enables the robot to perform natural, human-like movements, but also allows modulating them in amplitude, frequency or phase by modifying the dynamical system's parameters [10, 11].

The phase and frequency of an AFO's limit cycle are altered whenever the oscillator is coupled to an external dynamical system. Thus an interesting research question is whether an AFO-driven controller has the capacity to stabilize a dynamic system that is naturally unstable. In this paper we address that question in the context of controlling a bipedal walker. The walker analyzed here constitutes the simplest embodiment of the "limit cycle walking" paradigm, in which the walker tends towards a nominal periodic trajectory over the course of multiple steps, even though the trajectory is locally uncontrollable most of the time [12]. This class of walkers follows the principle of exploiting the natural dynamics of the bipedal walk, in particular the pendulum-like behavior of the swing leg, and has been shown to be extremely efficient from an energetic point of view $[13,14]$.

\footnotetext{
${ }^{*}$ School of Electrical, Mechanical and Mechatronic Systems, University of Technology, Sydney, Broadway, NSW 2007, Australia (email: gabriel.aguirre-ollinger@uts.edu.au).
} 
Limit cycle walking relaxes the requisite for continuous static stability which is at the core of zero-moment point (ZMP) control [15]. This greatly reduces the demand for actuator output but makes the walker more challenging to stabilize. Passive dynamic walking gaits typically exhibit very narrow domains of attraction and are therefore highly sensitive to perturbations [16, 17]. Different strategies have been proposed for increasing a dynamic biped's capability for disturbance rejection. These include controlling the placement of the leading leg before foot impact using a spring-like constraint [18] and retracting the swing leg before impact [19]. These methods have led to the implementation of successful walking prototypes.

We developed an AFO-based algorithm for the control of the simplest limit-cycle walker [13]. The algorithm uses a single AFO forming a closed feedback loop with the walker mechanism. The resulting system is capable of performing a periodic, highly stable gait cycle of which the stride frequency and step length can be tuned by adjusting the control gains. The AFO-based control drives the walker by means of hip-joint torques. The torque profiles and their timing are controlled by the phase of the AFO. A form of adaptive Fourier analysis [11] is employed to make the AFO instantaneous frequency as uniform as possible.

Our walking control method distinguishes between two phases of the gait cycle: stance, in which the leg is in contact with the ground, and swing, in which the leg has unconstrained movement. A specific control law is applied to each phase. In selecting these laws, we have sought to make the hip torques reasonably similar to the profiles generated by the hip-joint muscle groups in humans. These torque profiles, combined with the controller's stabilizing properties, are intended to make the method suitable for driving not only autonomous bipedal robots, but also powered exoskeletons and similar assistive devices for the human lower extremities.

Section 2 introduces the concept of limit cycle walkers and their global stability, and presents the dynamic walking model with hip actuation that constitutes the focus of this research. As a preparatory study for the control of the bipedal walker, section 3 analyzes the feedback control of an inverted pendulum using an AFO. The describing function method is employed to predict the existence of limit cycles in the closed-system and to determine the effect of the system's parameters on the amplitude and phase of the limit cycle. The AFO-based control of the dynamic walker model is formulated in section 4. The AFO uses the inter-leg separation angle as its input signal. Key features of the control method include the use of adaptive Fourier analysis to generate a uniform oscillator phase, and of a virtual spring with a movable equilibrium point to achieve gait stability. Section 5 presents a method for deriving gait solutions for the closed-loop dynamic walker, and analyzes the properties of the feasible walking solutions as functions of the control parameters. In that section we also analyze the orbital stability of the walker, which is indicative of the walker's ability to reject disturbances. Section 6 presents a study of the global stability of the walker which includes an algorithm for generating the basin of attraction of a particular walking solution. Finally, section 7 offers an initial study on the implementation of AFO-based control on a bipedal walker with a more realistic mass distribution and articulated knee joints. Potential stability issues are identified and their implications for control design are discussed.

\section{Dynamic walking model with hip actuation}

A stable limit cycle is an isolated, closed trajectory in state space to which neighboring trajectories converge. A limit-cycle walker utilizes the fact that the gait cycle is stable when observed at a 'landmark' state, even when the system is not locally stable or even locally controllable for the rest of the trajectory [12]. Thus a stable gait cycle can be represented as a Poincaré return map. The walker's forward motion maps the landmark state of the $k$-th stride $\mathbf{z}_{k}^{+}$ (state after leading foot impact) to a new state $\mathbf{z}_{k+1}^{+}$after one step: $\mathbf{z}_{k+1}^{+}=\mathbf{f}\left(\mathbf{z}_{k}^{+}\right)$. A periodic gait cycle exists if the walkers' landmark state is exactly repeated after one step: $\mathbf{z}^{*}=\mathbf{f}\left(\mathbf{z}^{*}\right)$, where $\mathbf{z}^{*}$ is known as a fixed point.

The orbital stability of the gait cycle can be determined by linearizing the stride function $\mathbf{f}$ about $\mathbf{z}^{*}$. The gait solution $\mathbf{z}^{*}$ is stable if the eigenvalues of the Jacobian of the stride function are contained in the unit circle in the complex plane. However, the linearized model only guarantees stability for small deviations from the gait solution. A global stability analysis is required to find the complete range of initial conditions, i.e. initial landmark states $\mathbf{z}_{0}^{+}$ from which the walking model can reach a steady gait cycle instead of falling down. If, for instance, one chooses the initial instant of leg swing as the walker's landmark state, the initial condition is defined by the angular positions and angular velocities of the walker's legs at that state. For a known periodic gait cycle, the entire set of initial conditions 


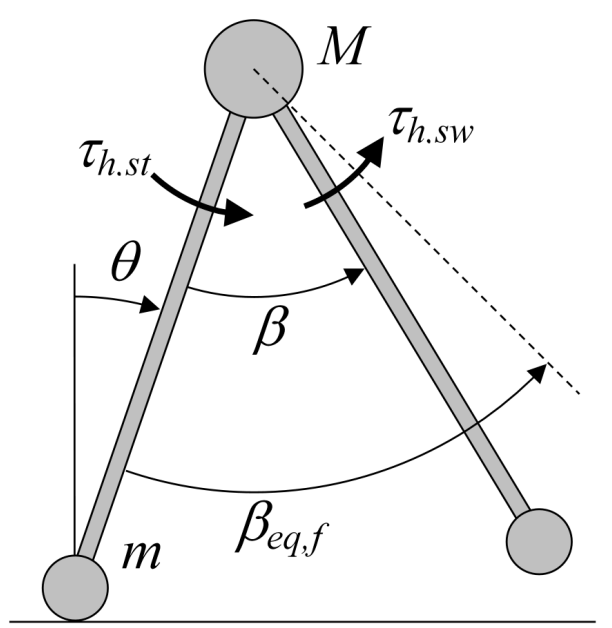

(a)

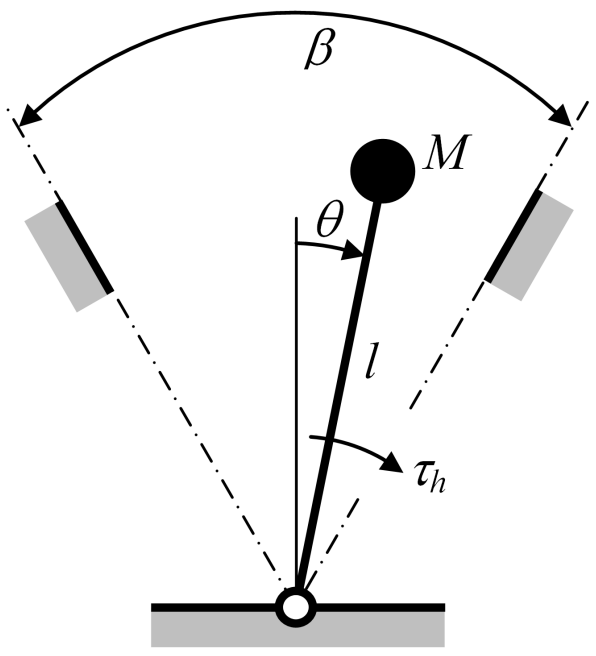

(b)

Figure 1: (a) Simplest dynamic walker. Condition $m / M \rightarrow 0$ decouples the motions of the stance leg and the swing leg. The pose of the stance leg is defined by the absolute angle $\theta$; the pose of the swing leg is defined by the inter-leg aperture angle $\beta$. The model is propelled by a hip torque $\tau_{h, s t}$ acting on the stance leg. A torque $\tau_{h, s w}$ drives the swing leg toward a desired separation angle $\beta_{e q, f}$ before striking the ground. (b) Inverted-pendulum model of the dynamic walker. The torque $\tau_{h}$ acting on the pendulum is equivalent to the stance leg torque $\tau_{h, s t}$ on the dynamic walker. Using the assumption that the swing leg does not perturb the trajectory of the stance leg, we replace the stance leg and the torque $\tau_{h, s w}$ with a pair of virtual walls, each on one side of the pendulum. The impact of the pendulum on the virtual wall is equivalent to the foot's impact on the ground.

that lead to it is known as the basin of attraction. In this paper we present an efficient method for computing the basin of attraction of the dynamic walker, based on the cell mapping method developed by Wisse et al. [18].

The starting point of our dynamic walker is the simplest walking model (SWM) studied by Garcia [16] and Kuo [13]. The SWM, represented in Figure 1(a) is essentially a two-link mechanism with point masses located at the "hip" joint and the feet. Per the simplification proposed by Garcia [16], in the equation of motion of the stance leg the ratio of foot mass to hip mass $(\mathrm{m} / M)$ tends to zero. This limit case is not to be understood as making the feet massless. Instead, it represents a condition in which the swing leg is unable to perturb the trajectory of the hip mass. In this way, the movements of the walker's legs are decoupled from each other.

A periodic gait cycle can be achieved on the SWM by a hip torque acting on the stance leg [13]. This torque adds momentum to the hip mass in order to replace the momentum transferred to the ground at foot impact. Actuating the swing leg, on the other hand, has no effect on the forward propulsion of the walker since the leg's mass is negligible compared to that of the hip. However, actuation of the swing leg can be employed to tune the stride frequency [13] and to enhance gait stability [12]. The scaled equations of motion of the SWM under the assumption of negligible mass of the swing leg are

$$
\begin{aligned}
\ddot{\theta}-\sin \theta & =-\tau_{h, s t} \\
\ddot{\beta}-\ddot{\theta}-\sin \beta \dot{\theta}^{2}+\sin \beta \cos \theta & =-\tau_{h, s w}
\end{aligned}
$$

At the end of the swing phase, the impact of the leading foot produces an instantaneous change in angular velocity. Conservation of angular momentum leads to a set of transition equations that yield the initial conditions for the next step [16]. 


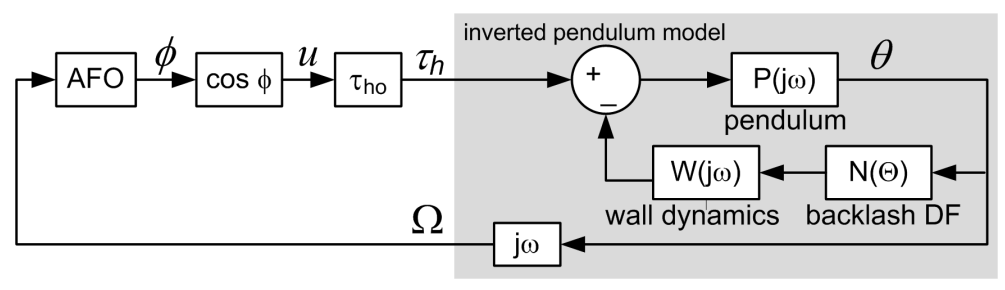

Figure 2: Block diagram of the inverted pendulum model driven by an AFO-generated torque $\tau_{h}$. The input to the backlash nonlinearity is the pendulum angle $\theta(t)$, which is assumed to be nearly sinusoidal.

\section{Gait control based on adaptive frequency oscillators: inverted-pendulum model}

A central pattern generator $(\mathrm{CPG})$ is a distributed biological neural network which can produce coordinated rhythmic signals without input from the brain or from sensory feedback [20]. Models of CPG's have been used to control the locomotion of autonomous robots [21, 10]. In this study we developed a method for bipedal walking control using an adaptive frequency oscillator (AFO) to perform the role of a CPG. An AFO can adapt in phase and frequency to an external input even when there is a large difference between the oscillator's initial frequency and the input frequency [7].

In this section we show how an AFO can generate a stable limit cycle when coupled to a simplified, quasilinear model of the bipedal walker. This model allows finding stable fixed points for the oscillation frequency $\omega$ analytically. The model also provides useful insights as to which parameters of the closed-loop AFO-walker system determine the final oscillation frequency.

A study by Buchli [21] analyzed an AFO forming a feedback loop with a linear-time-invariant (LTI) system. The system was shown to converge to a limit cycle with a frequency equivalent to the natural frequency of the LTI system; this frequency adaptation was described as 'finding resonance'. The notion of resonance tuning has also been applied to a CPG-driven bipedal walker to signify that the step frequency is proportional to the pendulum frequency of the swing leg [3]. However, as we will show, the AFO allows generating stable gait cycles for a wide range of frequencies than rather than just the natural frequency..

The quasi-linear model represents the lumped mass and the stance leg of the walker as an inverted pendulum in Figure 1(b). The AFO driving the pendulum is defined by the dynamical system

$$
\begin{aligned}
& \dot{\phi}=\omega-\epsilon \Omega(t) \sin \phi \\
& \dot{\omega}=-\epsilon \Omega(t) \sin \phi
\end{aligned}
$$

where $\phi$ is the oscillator phase, $\omega$ is the oscillator's intrinsic (but adaptable) frequency and $\Omega(t)$ is the angular velocity of the pendulum. The torque $\tau_{h}$ acting on the pendulum is proportional to the AFO output $\cos \phi$. The coupling strength $\epsilon$ determines the rate of adaptation of $\omega$ to the frequency of the input $\Omega(t)$. A virtual wall on each side of the pendulum provides a crude approximation of the foot's impact on the ground. The walls are placed at a constant angle of separation $\beta$. Choosing an arbitrary $\beta$ is consistent with the fact that, in the SWM, the motion swing leg is independent from that of the stance leg.

We model the separation between the virtual walls as a backlash nonlinearity. Limit cycles in a dynamic system with backlash can be predicted using the describing function method [22]. A describing function is a complex function defining the change in amplitude and phase of the nonlinearity's output relative to its input. In this method, the response of the nonlinear element to a sinusoidal input is treated as a Fourier series, but only the fundamental component of the output signal is considered in the model. This assumption is justified if the linear part of the system has good low-pass filtering characteristics. To satisfy the low-pass requirement, we have modeled the virtual walls as a linear spring-damper combination with a low stiffness coefficient. Damping also models the kinetic energy loss in the walker at ground strike.

The closed-loop inverted pendulum model is represented in block-diagram form in Figure 2. The output of the AFO, $u(t)$, is multiplied by a gain $\tau_{h o}$ (specified in units of torque) to generate the torque acting on the pendulum, $\tau_{h}(t)$. The input to the backlash nonlinearity is the pendulum angle $\theta(t)$, which is analogous to the absolute angle 


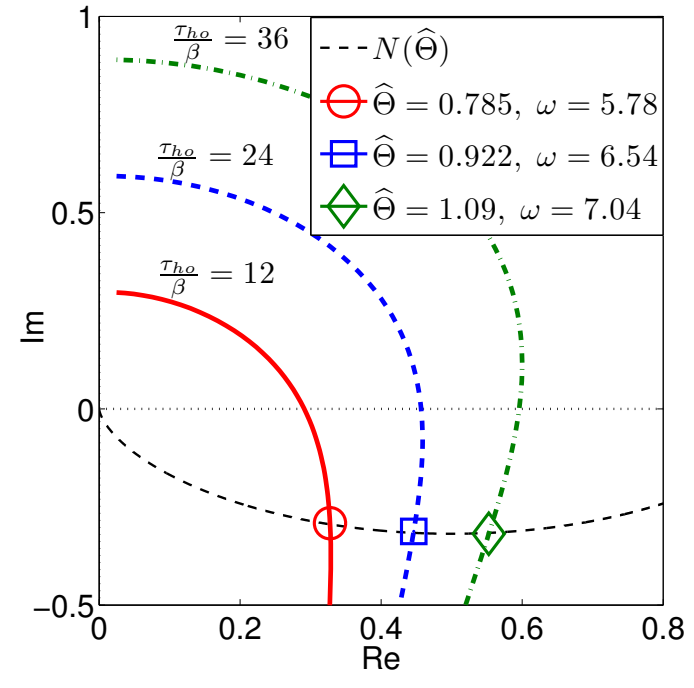

(a)

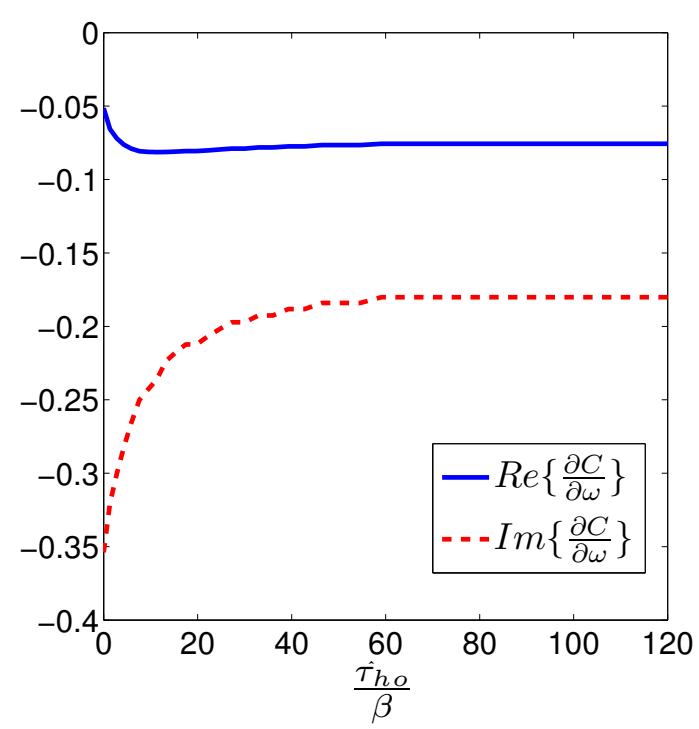

(b)

Figure 3: Limit cycles of the inverted pendulum model with backlash describing function. (a) Normalized limit cycle solutions $(\widehat{\Theta}, \omega)$ as a function of parameter $\tau_{h o} / \beta$. (b) Gradients of the pendulum model function $\frac{\partial C(\widehat{\Theta}, \omega)}{\partial \omega}$ as a function of $\tau_{h o} / \beta$. Parameter values: coupling $\epsilon=5, \omega_{n}=2 \pi, \zeta=1$.

of the stance leg in the walker. We assume this input to be the sinusoid $\theta(t)=\Theta \cos \omega t$. The problem of finding a limit cycle solution is equivalent to finding a nontrivial solution $(\Theta, \omega)$ to the system's characteristic equation

$$
C(\widehat{\Theta}, \omega) \equiv N(\widehat{\Theta})-Q(\widehat{\Theta}, \omega)=0
$$

where $\widehat{\Theta}=\Theta / \beta>1 / 2, N(\widehat{\Theta})$ is the backlash describing function and $Q(\widehat{\Theta}, \omega)$ is another complex function modeling the rest of the system's dynamics. The backlash describing function is given by

$$
\begin{aligned}
& \operatorname{Re}\{N(\widehat{\Theta})\}=\frac{1}{\pi}\left(\frac{\pi}{2}+B(\widehat{\Theta})+\frac{1}{2} \sin 2 B(\widehat{\Theta})\right) \\
& \operatorname{Im}\{N(\widehat{\Theta})\}=-\frac{\cos ^{2} B(\widehat{\Theta})}{\pi}
\end{aligned}
$$

where $B(\widehat{\Theta})=\sin ^{-1}\left(1-\widehat{\Theta}^{-1}\right)$. The function $Q(\widehat{\Theta}, \omega)$ (see derivation in Appendix A) is given by

$$
\begin{aligned}
& \operatorname{Re}\{Q(\widehat{\Theta}, \omega)\}=\frac{k\left(\omega^{2}+1\right)+\left(\frac{\tau_{h o}}{\beta}\right) \widehat{\Theta}^{-1} c \omega}{c^{2} \omega^{2}+k^{2}} \\
& \operatorname{Im}\{Q(\widehat{\Theta}, \omega)\}=\frac{-c \omega\left(\omega^{2}+1\right)+\left(\frac{\tau_{h o}}{\beta}\right) \widehat{\Theta}^{-1} k}{c^{2} \omega^{2}+k^{2}}
\end{aligned}
$$

Figure 3(a) shows limit cycle solutions $(\widehat{\Theta}, \omega)$ for (5). The main finding is that, unlike the AFO-coupled LTI system referred to earlier [21], here the frequency of the limit cycle is not determined by the intrinsic dynamics of the system. Instead, frequency can be modulated by the independent parameters $\tau_{h o}$ and $\beta$. In terms of walking, the fact that $\omega$ grows monotonically with $\tau_{h o} / \beta$ suggests that the walker's step frequency (which is commensurate with $\omega$ ) could be increased by simply increasing the amplitude of the torque on the stance leg. On the other hand, the same relationship suggests that, for a fixed torque amplitude, there may be an inverse relationship between step frequency and step length, the latter being proportional to $\beta$. 


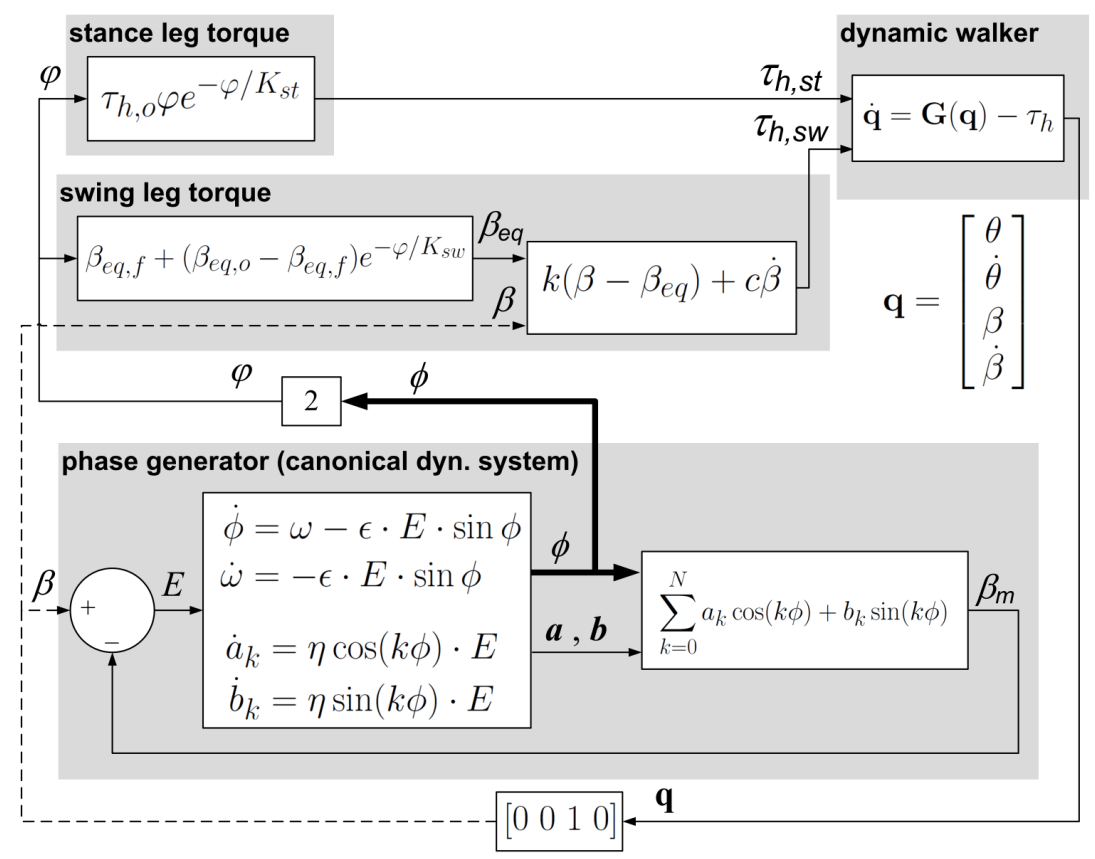

Figure 4: Model of the complete dynamic walker control. The phase generator employs a canonical dynamical system to perform an on-line Fourier analysis of the error signal $E$, and extract the phase $\phi$ of the fundamental frequency component. Hip torques are indexed to the phase of an individual stride, given by $\varphi=2 \phi$.

Figure 3(b) shows that the gradient $\frac{\partial C(\widehat{\Theta}, \omega)}{\partial \omega}$ has negative real and imaginary parts for a wide range of values of $\tau_{h o} / \beta$, indicating that the limit cycle solutions for the inverted pendulum have orbital stability. Thus the AFO can in principle generate a locally stable gait cycle in the bipedal walker, provided that the motion of the swing leg is effectively independent from that of the stance leg.

\section{Bipedal gait control driven by an adaptive frequency oscillator}

\subsection{Adaptive Fourier analysis using AFOs}

We present now an AFO-driven feedback controller for the complete dynamic walker. Controlling the relative phase of the legs' movement is key to achieving a stable gait [4]. Our dynamic walker control accomplishes this by linking the swing and stance torques to a reference phase $\phi(t)$. The "phase generator" takes the inter-leg separation angle $\beta$ and performs an on-line frequency analysis to extract its fundamental frequency and phase component. The complete model of the walker, featuring the phase generator and the torque profile functions is shown in Figure 4.

The phase generator is based on the canonical dynamical system proposed by Petric et al. [11]. A single AFO is combined with a feedback structure that performs an adaptive, on-line Fourier analysis. The AFO phase $\phi$ tracks the phase of the fundamental component of the error signal $E=\beta-\beta_{m}$, where $\beta$ is the measured inter-leg angle and $\beta_{m}$ is the reconstructed inter-leg angle, given by the Fourier series

$$
\beta_{m}=\sum_{k=0}^{N} a_{k} \cos (k \phi)+b_{k} \sin (k \phi)
$$

In (10), $N$ is the number of components of the Fourier series and $a_{k}$ and $b_{k}$ are the Fourier components' amplitudes, which are governed by the adaptation laws 


$$
\begin{aligned}
& \dot{a}_{k}=\eta \cos (k \phi) E \\
& \dot{b}_{k}=\eta \sin (k \phi) E
\end{aligned}
$$

where $\eta$ is a learning constant. The role of (10) in our control method is only as a filter to make the slope of $\phi$ as uniform as possible; the walker could in principle be driven by the AFO alone.

\subsection{Model of hip-joint torque during stance}

Our dynamic walker uses a simple bell-shaped motor primitive to imitate the burst-like behavior of the hip extensor muscles during walking [23]. To control the timing of the burst, the torque profile is linked to the phase of one single stride. Because the AFO phase $\phi$ completes one cycle ( $\phi=0$ to $2 \pi$ ) for every two strides, the stride phase is given by $\varphi=2 \phi$. The stance torque profile is defined in terms of $\varphi$ as

$$
\tau_{h, s t}=\tau_{h, o} \varphi e^{-\varphi / K_{s t}}
$$

Thus the amplitude and decay rate of the torque profile are controlled by the parameters $\tau_{h o}$ and $K_{s t}$ respectively.

\subsection{Spring-damper torque for swing leg with traveling equilibrium point}

The stabilization strategy follows the principle formulated by Wisse [18] of guaranteeing that the swing leg will strike the ground at the proper leg separation angle $\beta$. This can be accomplished by generating a virtual spring with a traveling equilibrium point. In this scheme the initial equilibrium of the spring coincides with the initial position of the swing leg. The equilibrium point $\beta_{e q}$ travels from an initial position $\beta_{e q, o}$ to a final position $\beta_{e q, f}$ following a smooth trajectory controlled by $\varphi$ :

$$
\tau_{h, s w}=\kappa\left(\beta-\beta_{e q}(\varphi)\right)+\nu \dot{\beta}
$$

where $\beta_{e q}(\varphi)=\beta_{e q, f}+\left(\beta_{e q, o}-\beta_{e q, f}\right) e^{-\varphi / K_{s w}}$ and $K_{s w}$ controls the rate at which the equilibrium point converges to its final value. The term $\kappa$ in (14) is the virtual spring constant. A torsional damping term with coefficient $\nu=2 \sqrt{\kappa}$ is included to ensure that the swing leg behaves as a critically-damped system. This prevents the leg from oscillating about the equilibrium point before foot strike.

In the course of a walking stride, the hip torque profiles (13) and (14) are applied simultaneously to the corresponding legs. Then, at foot impact, the swing leg becomes the stance leg for the next stride and viceversa. The control model assumes that some form of foot-impact detection is available in order to switch the torque profiles among the legs.

\section{Gait solutions for the dynamic walker}

The gait cycle of the dynamic walker is represented as a Poincaré return map from the walker's state after foot impact. This state combines both the walker's independent kinematic variables $(\theta, \dot{\theta})$ and the AFO state variables

$$
\mathbf{z}^{+}=\left[\theta^{+} \dot{\theta}^{+} \phi^{+} \omega^{+}\right]^{T}
$$

Thus a stable gait corresponds to a fixed point $\mathbf{z}^{*}=\mathbf{f}\left(\mathbf{z}^{*}\right)$. In this section we present walking solutions (fixed points) for the dynamic walker. Our focus is the direct kinematics problem, i.e. to find, for a certain combination of control parameters $\left(\tau_{h o}, \beta_{e q, f}\right)$ and AFO parameters, the landmark state $\mathbf{z}^{*}$ that defines the walking solution.

Searching for solutions via an optimization algorithm is unlikely to succeed due to the inherent instability of the walker mechanism. Because a large proportion of the possible initial conditions will cause the walker to fall down in simulation, in general it is not possible to generate a smooth cost function for the optimization. Instead, we used a linearized model of the AFO-driven walker model to obtain an approximate gait solution, and employed that 
(a)

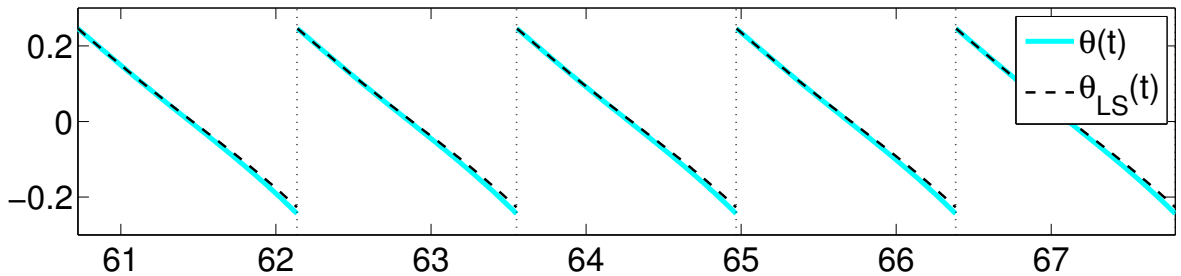

(b)

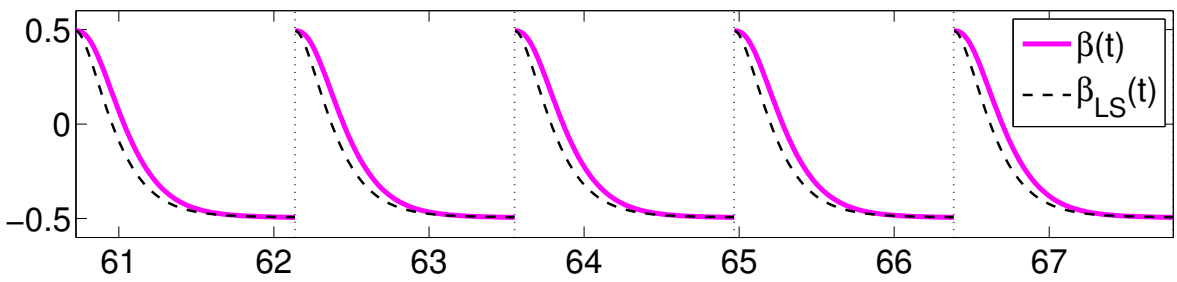

(c)

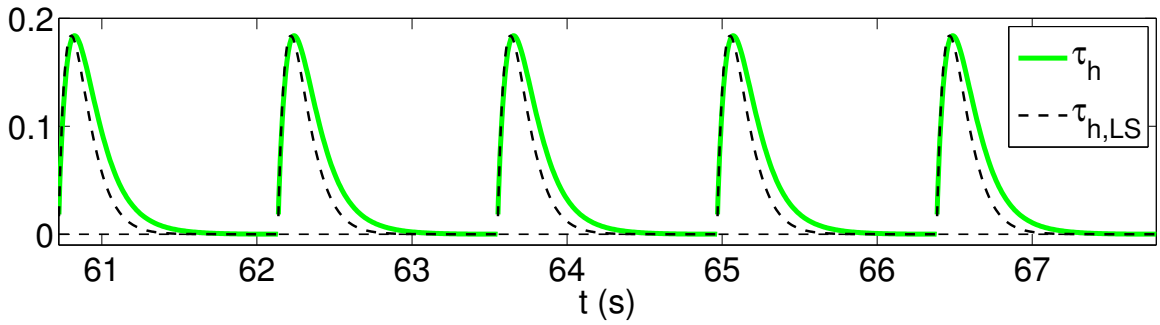

Figure 5: Time plots for gait solutions. A gait solution is considered to be valid if the walker can execute 100 steps in simulation without falling down. Plots show only a portion of the simulation starting at about $61 \mathrm{~s}$; it is assumed that by this time the walker has reached a uniform gait cycle (fixed point). In these plots the time trajectories of the linearized model (subscript 'LS') are compared against those of the original, nonlinear walking model. (a) stance leg angle $\theta$,(b) angle of separation between legs $\beta$ and (c) hip torque acting on the stance leg, $\tau_{h}$. The following parameter values were used for the simulation: coupling parameter $\epsilon=10$, learning constant $\eta=8$, stance leg torque gain $\tau_{h o}=1$ and final equilibrium angle $\beta_{e q, f}=-0.5$.

solution as the initial condition for simulating the gait of the original nonlinear walker. The underlying assumption was that, if the linearized solution was sufficiently close to an actual fixed point, orbital stability would allow the nonlinear walker to reach the fixed point after a certain number of strides.

The derivation of the linearized walking model is presented in Appendix B. Aside from the linearization of the walker dynamics, the other major assumption is that the AFO has already converged to a periodic limit cycle, and thus the AFO frequency $\omega$ can be treated as constant. The adjustable parameters of the linearized model are the hip torque amplitude $\tau_{h o}$ and the spring equilibrium point $\beta_{e q}=\beta_{e q, f}$. Equations (68), (70) and (72) in Appendix B can be solved numerically to yield the approximate gait parameters $\Theta, \Omega$ and $T_{s}$. These parameters are then employed as initial conditions for a simulation of the original closed-loop walking model:

$$
\begin{aligned}
& \theta^{+}(0)=\Theta \\
& \dot{\theta}^{+}(0)=-\Omega \\
& \phi(0)=0 \\
& \omega(0)=\pi / T_{s}
\end{aligned}
$$

Figure 5 shows a comparison between simulations of the original walker model and its linearized version. The plots illustrate how agreement between the behavior of models can be quite high as long as the walking solution from the linearized model effectively converges to a fixed point of its nonlinear counterpart. It should be noted that, since this is a rigid-leg model, there is no compliance involved in foot impact, and as a result the walking gait does not have a finite-time double-support phase. Thus the transfer of momentum from the walker to the ground is 
(a) AFO output $\left(\varepsilon=10, \mathrm{k}=50, \tau_{\mathrm{ho}}=4, \beta_{\text {eq, }}=-0.7, \eta=8\right)$

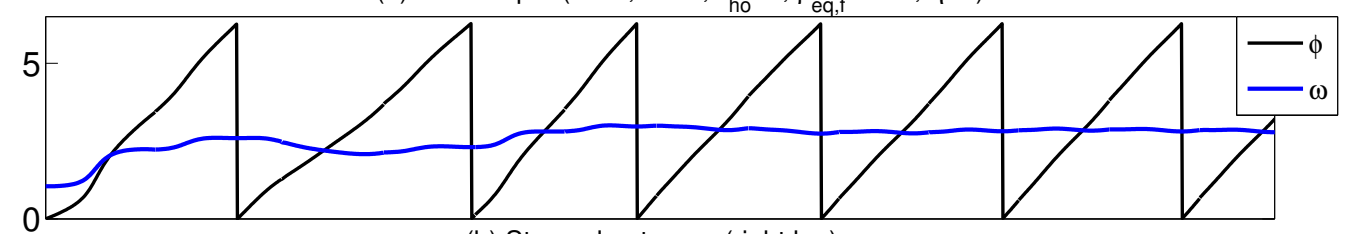

(b) Stance leg torque (right leg)

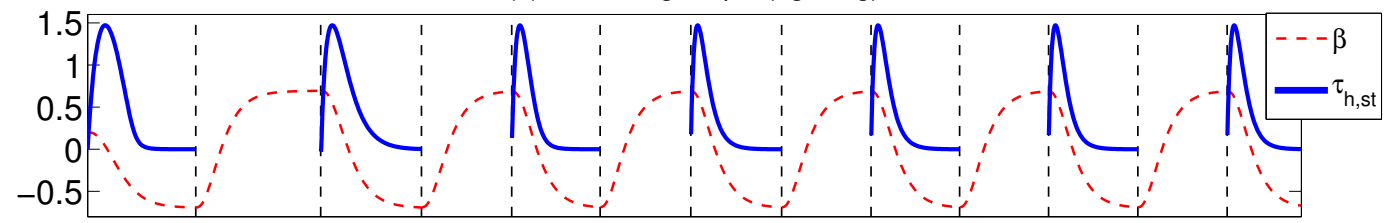

(c) Swing torque (right leg)

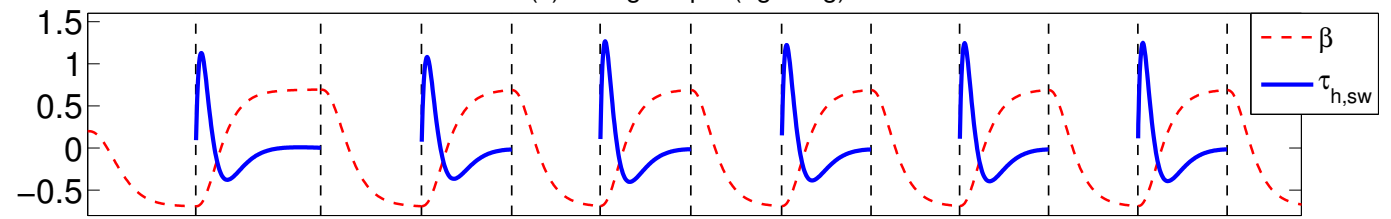

(d) Ground reaction force

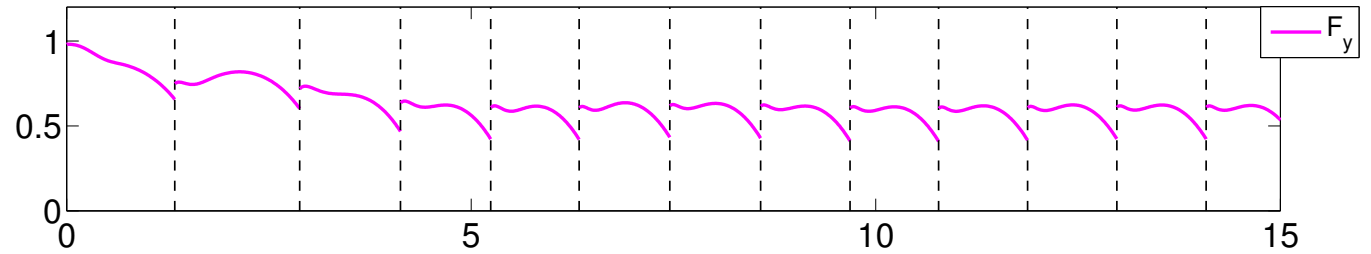

Figure 6: Simulation results for the dynamic walker with AFO-driven feedback control. Plots show example time trajectories of (a) AFO phase $\phi$ and intrinsic frequency $\omega$, (b) stance leg torque $\tau_{h, s t}$ (with inter-leg separation angle $\beta$ for reference), (c) swing leg torque $\tau_{h, s t}$ (ditto) and (d) ground reaction force $F_{y}$ on the stance foot. In order to make the slope of the phase as uniform as possible, the inter-leg angle $\beta$ is multiplied by -1 every other step, thereby ensuring a smooth transition. Simulation parameters: coupling parameter $\epsilon=10$, learning constant $\eta=8$, virtual spring constant $\kappa=50$, stance leg torque gain $\tau_{h o}=4$, final equilibrium angle $\beta_{\text {eq,f }}=-0.7$.

instantaneous.

We implemented the AFO-driven walking model in Matlab/Simulink (The Mathworks, Natick, MA, USA) to find walking solutions. Simulations employed a variable-step solver (ode45) with maximum step size of $0.01 \mathrm{~s}$. The walker is considered to have reached a fixed point when the magnitude of the difference between successive states is less than a certain error threshold: $\left\|\mathbf{z}_{k+1}^{+}-\mathbf{z}_{k}^{+}\right\|<e_{\mathbf{z}}$. The search for a fixed point is considered to fail if the walker either falls down in simulation or fails to reach the threshold condition before a predetermined number of steps $N_{s}$. For the present study we chose $N_{s}=100$.

Figure 6 shows an example of a gait simulation. The adaptation of the AFO frequency $\omega(t)$ to a uniform value is readily apparent. As $\omega(t)$ adapts, the hip torque profiles $\tau_{h, s t}(t)$ and $\tau_{h, s w}(t)$ and the ground reaction force $F_{y}(t)$ adopt a periodic behavior.

Figure 7 presents a map of solution points $\left(\theta^{*}, \dot{\theta}^{*}\right)$ for different combinations of $\tau_{h o}$ and $\beta_{e q, f}$. The selected values of coupling parameter $\epsilon=10$ and spring constant $\kappa=50$ yielded a fairly large array of gait solutions. In general, increasing $\tau_{h o}$ for a constant $\beta_{e q, f}$ increases the initial angular speed $\dot{\theta}^{*}$ while keeping the stride amplitude (determined by $\theta^{*}$ ) nearly constant. A point of interest is that the AFO-based closed-loop control can generate solutions outside the boundaries of the passive simplest walking model (SWM) [13, 18]. The passive SWM requires the initial kinetic energy after foot strike, $K^{+}=\frac{1}{2} \dot{\theta}^{2}$ (after normalization), to be greater than the change in potential energy required for the hip mass to "pole vault" over the stance foot, $\Delta P=1-\cos \theta$. This condition is represented by the boundary curve in Figure 7. An initial condition placed above the boundary will cause the passive walker to eventually fall backward. By contrast, the AFO-driven walker was able to generate walking solutions outside this 


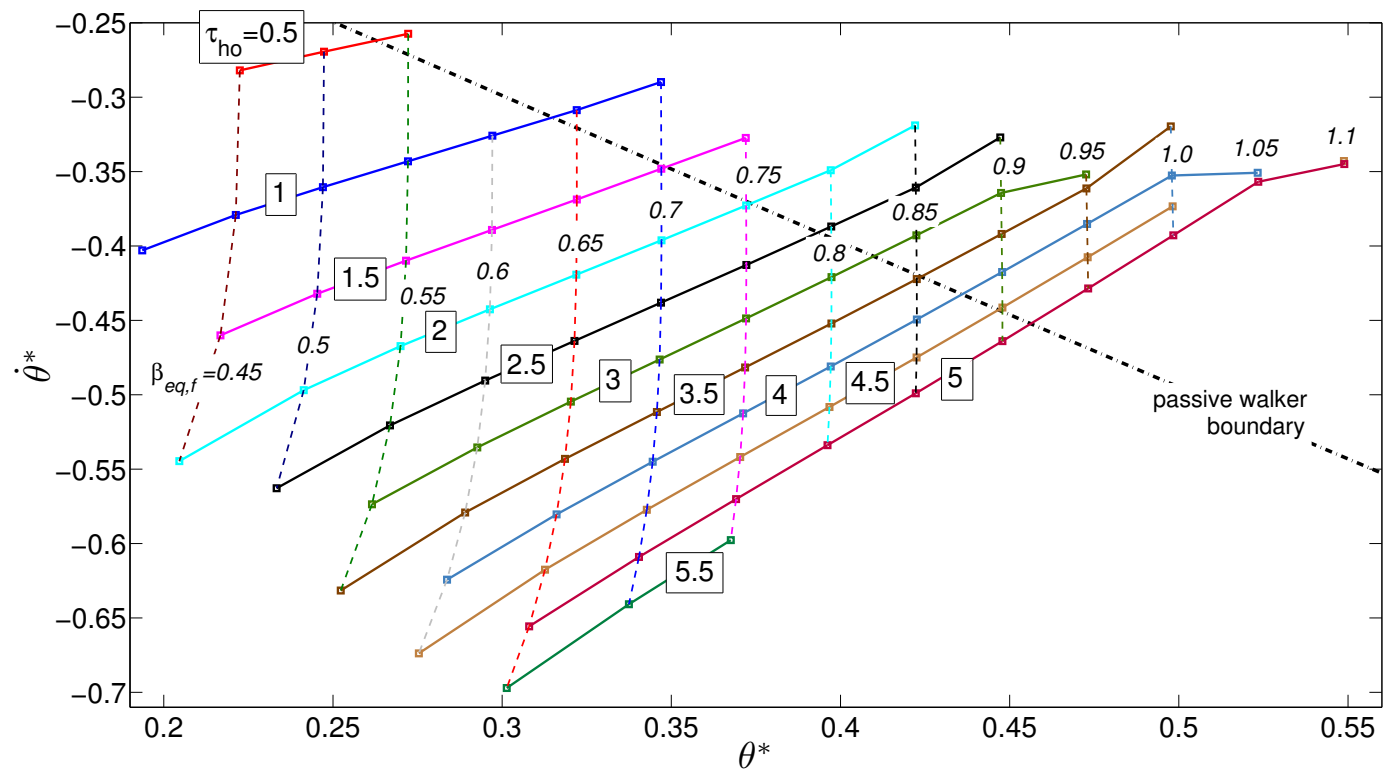

Figure 7: Walking solutions $\left(\theta^{*}, \dot{\theta}^{*}\right)$ map for $\epsilon=10$ and $k=50$. Solid curves represent approximate contours of constant hip torque gain $\tau_{h o}$. Values of $\tau_{h o}$ are shown in boxes. Dashed curves represent contours of constant spring equilibrium $\beta_{e q, f}$. Values of $\beta_{e q, f}$ are shown in italics. A number of solutions occurs outside the passive walking boundary.

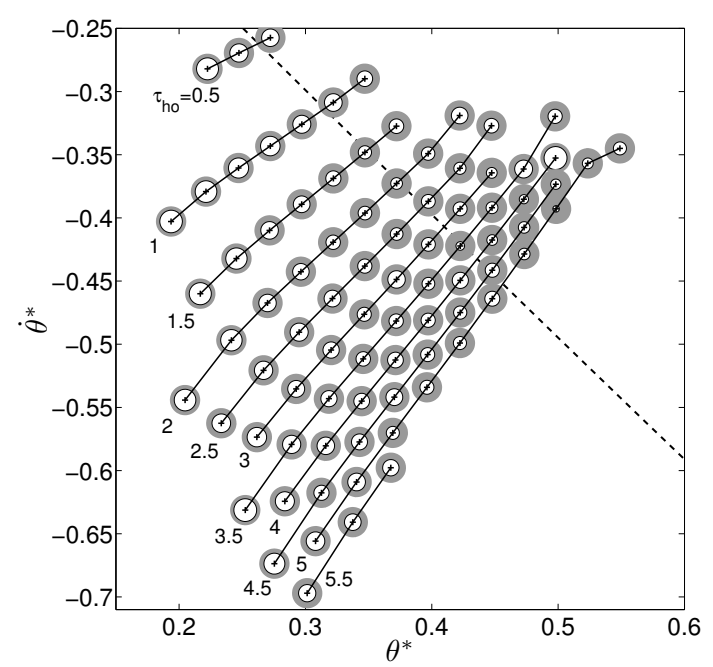

(a)

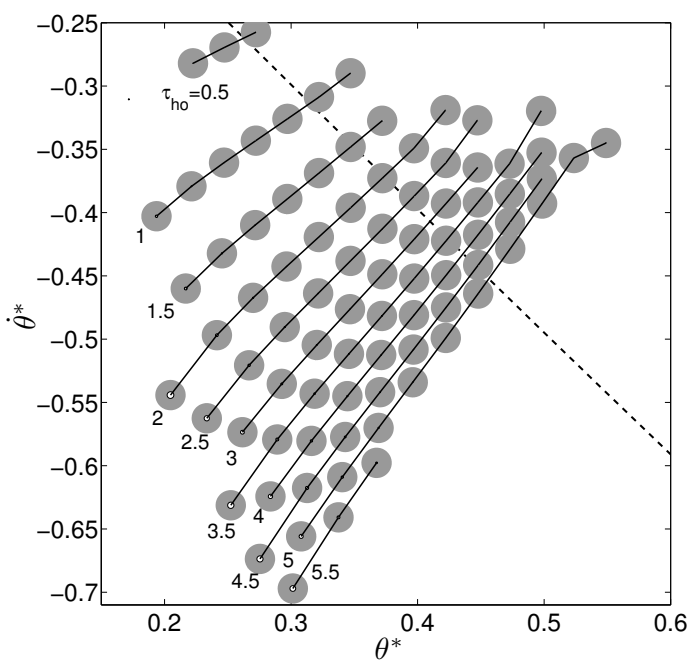

(b)

Figure 8: Eigenvalues of the return map Jacobians for different gait solutions $\left(\theta^{*}, \dot{\theta}^{*}\right)$. (a) In this plot the white disk centered at each solution point represents the magnitude of the maximum eigenvalue for $\left(\theta^{*}, \dot{\theta}^{*}\right)$. For reference, a gray disk represents the unit circle. Continuous curves represent contours of constant $\tau_{h o}$. (b) Maximum eigenvalue magnitudes in the absence of perturbation on $\dot{\theta}^{*}$. The majority of the eigenvalues become of near-zero magnitude, suggesting that the system is mostly sensitive to velocity perturbations.

boundary. 


\subsection{Orbital stability}

Orbital stability of the walker, i.e. stability to small deviations of the landmark state $\mathbf{z}^{+}$from the fixed-point value $\mathbf{z}^{*}$ can be determined from the Jacobian matrix of the return map,

$$
\mathbf{J}\left(\mathbf{z}^{*}\right)=\left.\frac{\partial \mathbf{f}(\mathbf{z})}{\partial \mathbf{z}}\right|_{\mathbf{z}=\mathbf{z}^{*}}
$$

A return map is stable for small perturbations of $\mathbf{z}^{*}$ if all the eigenvalues of its Jacobian are within the unit circle. The smaller the eigenvalues, the faster the walker will converge to the fixed point $\mathbf{z}^{*}$. The Jacobian $\mathbf{J}\left(\mathbf{z}^{*}\right)$ can be approximated numerically using a perturbation method. For each term $f_{i}(\mathbf{z})$ in $\mathbf{f}(\mathbf{z})$, the $k$-th column term in the Jacobian is

$$
J_{i, k}=\frac{\partial f_{i}}{\partial z_{k}} \simeq \frac{f_{i}\left(\mathbf{z}^{*}+\Delta \mathbf{z}_{k}\right)-f_{i}\left(\mathbf{z}^{*}\right)}{\Delta z_{k}}
$$

where $\Delta \mathbf{z}_{k}$ is a vector in which the $k$-th terms equals $\Delta z_{k}$ and the remaining terms are equal to zero.

Figure 8(a) shows the maximum eigenvalue magnitudes for the gait solutions previously derived. In all cases the eigenvalues were within the unit circle, indicating that, for moderate perturbations, the system will return to the fixed point after a certain number of steps. However, there is no clear correlation between the eigenvalues of a particular solution and its proximity to the boundaries of the solutions' region. Therefore the eigenvalues of the Jacobian are of limited use in predicting the size of the region of feasible walking solutions. On the other hand, the eigenvalues provide a measure of the sensitivity of the limit cycle to variations in the different state variables. The kinematic variable that most severely impacts the stability of the walker is the angular velocity $\dot{\theta}^{*}$ of the stance leg. This point is evidenced by obtaining the Jacobian for the case of zero perturbation in $\dot{\theta}^{*}$. As is shown in Figure 8(b) the eigenvalues become nearly zero for most of the walking solutions, indicating that the periodic limit cycle will recover quickly from perturbations to state variables other than $\dot{\theta}^{*}$.

For a more precise estimation of the walker's stability it is necessary to determine the global stability of each gait solution. In section 6 we present a fast algorithm for determining the approximate basin of attraction of a particular gait solution.

\subsection{Comparison to the inverted pendulum model with backlash}

The inverted pendulum model with backlash previously analyzed showed the frequency of the limit cycle to be proportional to the ratio $\tau_{h o} / \beta$ (Figure 3(a)). To show that the dynamic walker has the same qualitative behavior, we generated a set of gait solutions using the ratio $\tau_{h o} / \beta_{e q, f}$ as an adjustable parameter. From Figure 9(a) it can be inferred that step frequency (approximately $2 \omega^{*}$ ) is proportional to $\tau_{h o} / \beta_{e q, f}$ and varies inversely with stride length, which is typically nearly equal to $\beta_{e q, f}$. Figure 9(b) shows that the walker's average forward speed $v_{f}$ is quite uniform for constant $\tau_{h o} / \beta_{e q, f}$. This is due to the fact that $v_{f}^{*}, \omega^{*}$ and $\beta_{e q, f}$ are related by

$$
v_{f}^{*} \sim \omega^{*} \beta_{e q, f}
$$

and, from Figure 9(a), for constant $\tau_{h o} / \beta_{e q, f}$ there is an approximately inverse relationship between $\omega^{*}$ and $\beta_{e q, f}$.

\section{Global stability of the dynamic walker}

We present now a method for determining the global stability properties of the dynamic walker. The objective is to find, for a particular gait solution $\mathbf{z}^{*}$, the largest region of possible initial conditions from which the walker's gait will converge to the specified gait solution. A sequence of $k$ successive strides from an initial state $\mathbf{z}_{o}$ is represented as $\mathbf{z}_{k}=\mathbf{f}^{k}\left(\mathbf{z}_{o}\right)$. A periodic gait cycle $\mathbf{z}^{*}$ is defined by $\mathbf{z}^{*}=\mathbf{f}\left(\mathbf{z}^{*}\right)$. This gait cycle constitutes a globally stable fixed point if there exist a set $A\left(\mathbf{z}^{*}\right)$ of initial states in the vicinity of $\mathbf{z}^{*}$ such that the walker converges to $\mathbf{z}^{*}$ in a finite number of steps:

$$
A\left(\mathbf{z}^{*}\right)=\left\{\mathbf{z}_{o} \mid \mathbf{f}^{k}\left(\mathbf{z}_{o}\right)=\mathbf{z}^{*}\right\} \text { for } k \geq N_{s}
$$

where $N_{s}$ is a finite integer. We refer to $A$ as the basin of attraction of $\mathbf{z}^{*}$. 
(a)

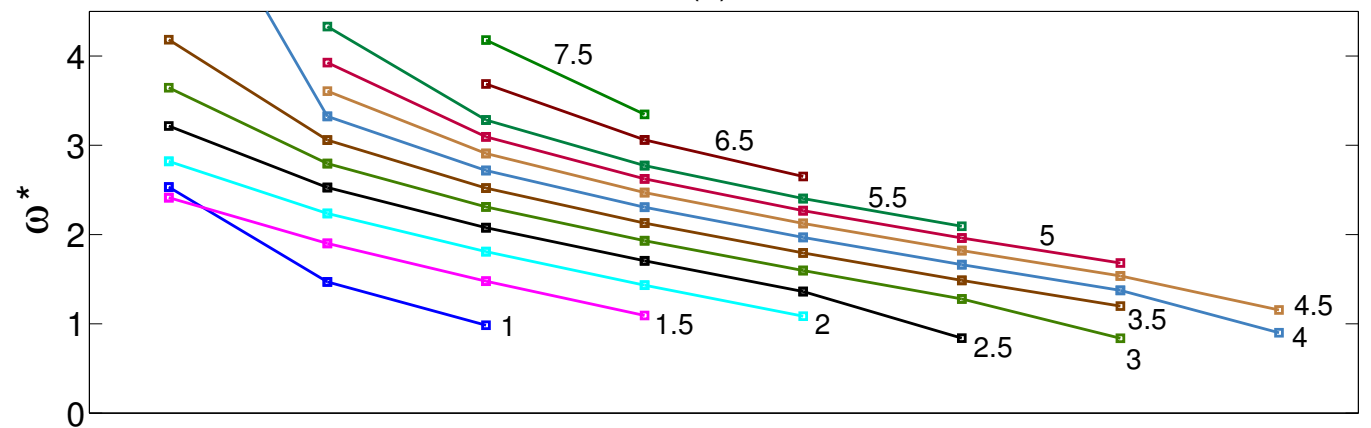

(b)

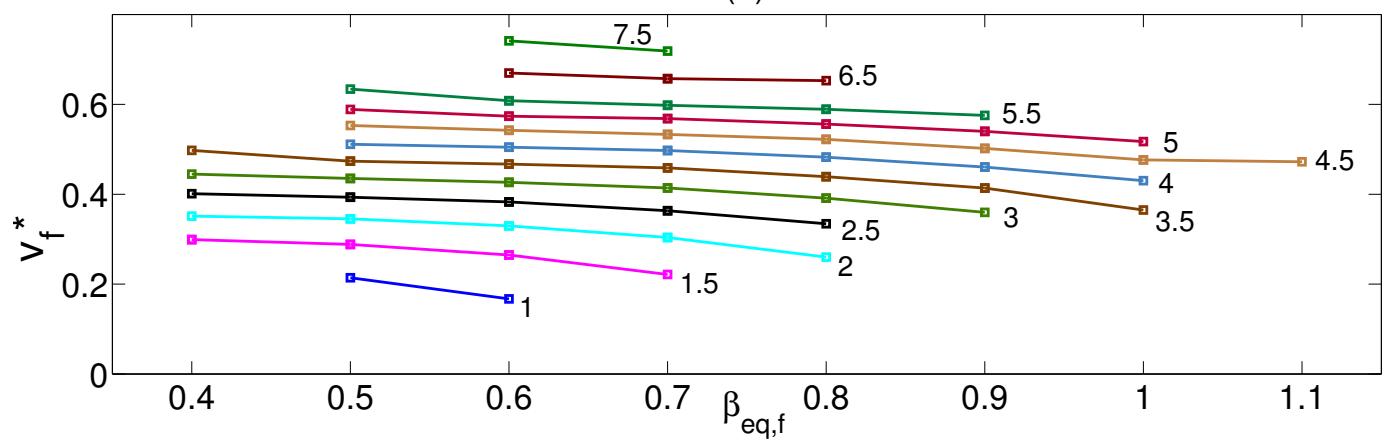

Figure 9: Frequency and forward speed of the gait solutions for the dynamic. Contour plots represent constant values of $\tau_{h o} / \beta_{e q, f}$. (a) AFO frequency $\omega^{*}$ vs. final spring equilibrium angle $\beta_{e q, f}$. (b) Walker forward speed $v_{f}^{*}$ vs. $\beta_{e q, f}$.

We obtain the approximate basin of attraction of the gait cycle $\mathbf{z}^{*}$ using a method similar to cell mapping [24, 17]. The region of possible initial states $\mathbf{z}_{o}$ is subdivided into discrete "cells" of interval size $\Delta \mathbf{z}$. A cell $\mathbf{c}$ is a vector of the same dimension as $\mathbf{z}$, composed of integer values $c_{i}$. A point $\mathbf{z}$ in state space is mapped to a cell $\mathbf{c}$ via the transformation

$$
\mathbf{c}=\Gamma(\mathbf{z}) \mid\left(c_{i}-1\right) \Delta z_{i} \leq z_{i}<c_{i} \Delta z_{i}
$$

The inverse transformation returns the center of the cell in state space coordinates, $\mathbf{z}_{c}$ :

$$
\mathbf{z}_{c}=\Gamma^{-1}(\mathbf{c}) \mid z_{c, i}=\left(c_{i}-\frac{1}{2}\right) \Delta z_{i}
$$

In general, to obtain the discretized basin of attraction, we use the center $\mathbf{z}_{c}$ of each cell as an initial state for the walker, and test whether the walking simulation converges to $\mathbf{z}^{*}$, i.e. whether the following sequence of states exists for the walker:

$$
Z\left(\mathbf{z}_{c}, \mathbf{z}^{*}\right)=\left\{\mathbf{z}(k) \mid \mathbf{z}(k)=\mathbf{f}^{k}\left(\mathbf{z}_{c}\right)=\mathbf{z}^{*} \text { for } k>N_{s}\right\}
$$

The discretized counterpart of (23) is a sequence of cells

$$
C\left(\mathbf{z}_{c}, \mathbf{z}^{*}\right)=\Gamma\left(Z\left(\mathbf{z}_{c}, \mathbf{z}^{*}\right)\right) \equiv\{\mathbf{c}(k)=\Gamma(\mathbf{z}(k)) \mid \mathbf{z}(k) \subset Z\}
$$

Thus the discretized basin of attraction is the set of all possible sequences of cells ending in $\mathbf{c}^{*}=\Gamma\left(\mathbf{z}^{*}\right)$ :

$$
A_{c}\left(\mathbf{z}^{*}\right)=\bigcup\left\{C\left(\mathbf{z}_{c}, \mathbf{z}^{*}\right)\right\}
$$

It would be computationally too expensive to generate the basin of attraction for all feasible initial states $c$ because $\mathbf{z}_{c}$ is 4-dimensional. Instead, we will employ a model of reduced dimensions by making a few simplifying assumptions. The first assumption is that the walking model always starts from same initial conditions for the AFO, namely $\phi(0)=\phi^{*}=0$ and $\omega(0)=\omega^{*} \simeq \pi / T_{s}$, where $T_{s}$ is the stride period of $\mathbf{z}^{*}$. This is reasonable because we 
(a)

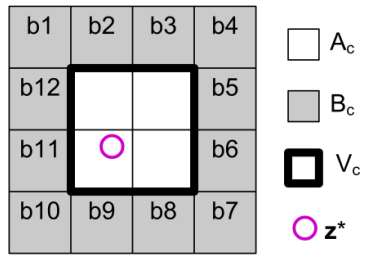

(d) (b)

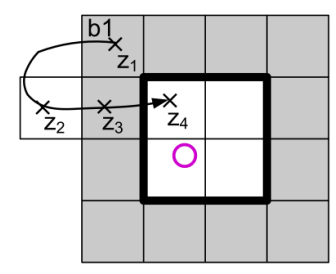

(c)

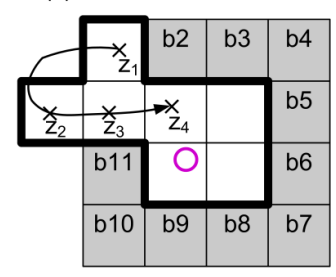

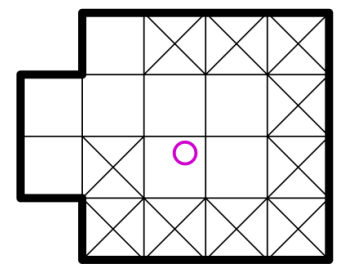

(e)

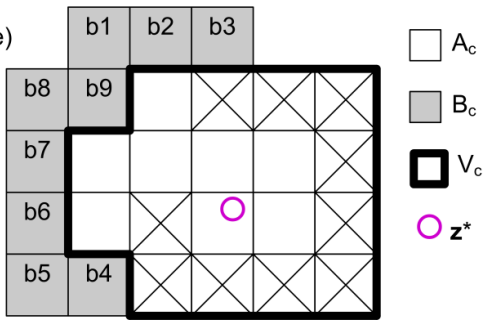

Figure 10: Generation of the basin of attraction using a fast algorithm with phase resetting. (a) The current basin $A_{c}$ includes one cell containing the gait solution $\mathbf{z}^{*}$. A boundary of cells $B_{c}$ is generated around $A_{c}$ by means of a dilation operator. (b) A walking simulation begins from an initial condition located at the center of a boundary cell ( $\mathbf{z}_{1}$, representing the center of $b_{1}$ in state space) and reaches a cell belonging to $A_{c}$. (c) All the cells visited in the preceding simulation are appended to $A_{c}$. The set $V_{s}$ of visited cells (thick border) is increased accordingly. (d) Basin of attraction $A_{c}$ and set of visited cells $V_{c}$ after all the boundary cells have been tested and removed from $B_{c}$. "Crossed-out" cells represent initial conditions that failed to generate a path into $A_{c}$. (e) A new boundary is generated around $A_{c}$, but excluding any previously visited cells $\left(V_{c}\right)$.

only intend to design for robustness to perturbations in the kinematic initial conditions, i.e. the initial values of $\theta$ and $\dot{\theta}$.

A bolder assumption is that every two strides the algorithm will reset the AFO phase and frequency to their fixed-point values, i.e. enforce $\phi^{+}=0$ and $\omega^{+}=\omega^{*}$. Resetting is applied precisely every two strides because, in a uniformly periodic gait, $\phi$ undergoes one cycle (i.e. goes from 0 to $2 \pi$ ) for every two strides. The Fourier coefficients are reset to their initial values as well. Thus the state space of the walker is reduced to a two-dimensional space. Moreover, in this way every cell in the discretized basin of attraction can function either as an initial condition, or as an intermediate state in a gait sequence that began elsewhere. The latter property allows implementing the algorithm in a recursive manner.

In our recursive algorithm, a cell is labeled a "visited" cell if it is either an initial state, or is arrived at via successive Poincaré mappings. The mapping sequence stops when the walker either fails to complete a stride (i.e. falls down) or reaches a previously visited cell. Initially $A_{c}$ contains only the cell $\mathbf{c}^{*}$. The algorithm recursively creates a border of cells $B_{c}$ around $A_{c}$ using a "dilation" morphological operator [25]. Each cell on $B_{c}$ is tested as an initial condition for walking. If the cell can successfully generate a path into $A_{c}$, then all the cells in the path are added to $A_{c}$. The algorithm is presented in pseudocode in Table 1, with Figure 10 as a reference.

The end condition numel $\left(A_{c}-A_{c, \text { old }}\right)=0$ is satisfied when the last boundary $B_{c}$ generated by the algorithm produces no new cells for the basin of attraction. Figure 11 shows the example of a basin of attraction for a particular gait solution $\left(\theta^{*}=0.29501\right.$ and $\left.\dot{\theta}^{*}=-0.49043\right)$ computed using the fast algorithm with phase resetting.

\section{Towards an AFO-driven gait control for a walker with multiple degrees of free- dom}

The next goal in this research is to extend the AFO-driven gait control to more complex walkers with multiple degrees of freedom (DOF). Coordination among the joint torques can be accomplished by making them time-invariant functions of the AFO phase $\phi$. This approach has the advantage of reducing the number of DOF of the system, 


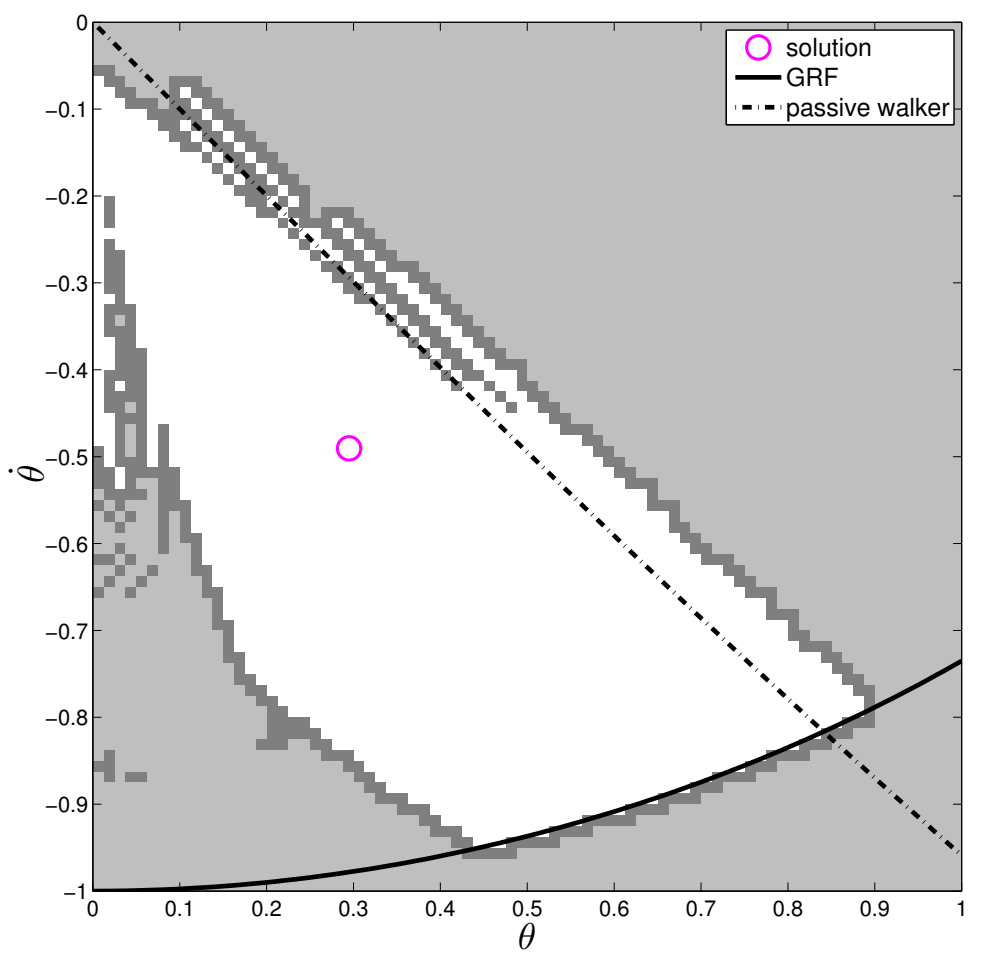

Figure 11: Basin of attraction (white cells) for a gait solution (fixed point) of the dynamic walker, using fast algorithm with resetting of the AFO phase and frequency. The gait solution is $\theta^{*}=0.29501$ and $\dot{\theta}^{*}=-0.49043$. The boundary (dark gray cells) is composed of initial conditions for which the walker's gait fails before reaching the fixed point. The curve labeled 'GRF' is the running boundary, beyond which the stance foot will break ground contact. The dashed curve represents the stability boundary for the passive SWM [17]. Simulation parameters: $\epsilon=10, \eta=8$, $k=50, \tau_{h o}=2.5, \beta_{e q, f}=-0.6$.

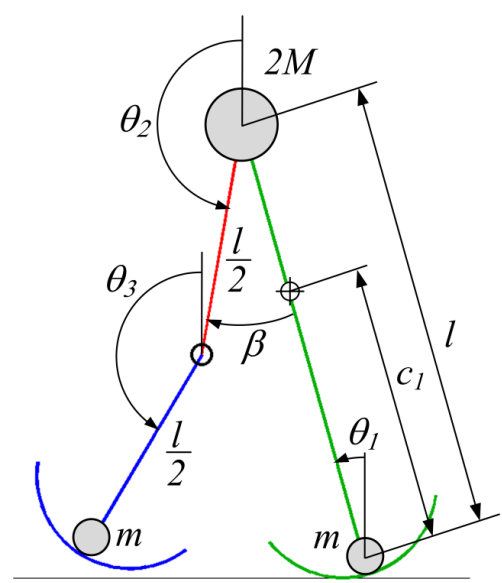

(a)

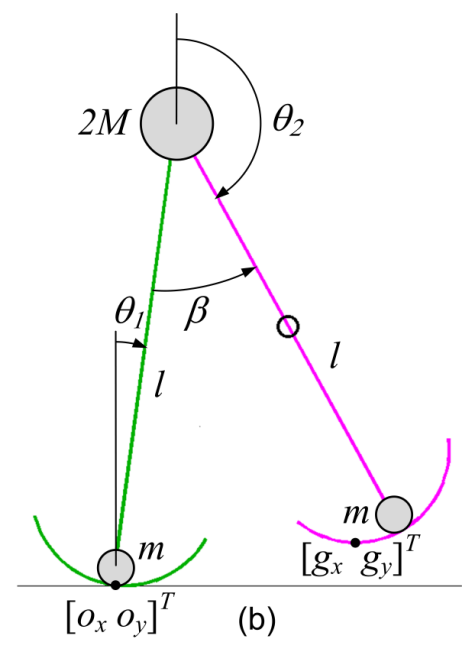

(b)

Figure 12: Biped walker diagrams.(a) Phase 1. (b) Phase 2.

effectively making the oscillator perform the role of a CPG. But in order to design the control for multiple joints it is important to understand the how the control's performance may change when applied to a bipedal walker with realistic dynamics. To this end we tested the AFO on a dynamic walking model that features articulated knee joints and a finite ratio of hip to foot mass. The objective was to determine whether the stability and gait properties of the system would be affected by (1) the swing leg perturbing the trajectory of the stance leg given the finite mass ratio, 
(a)

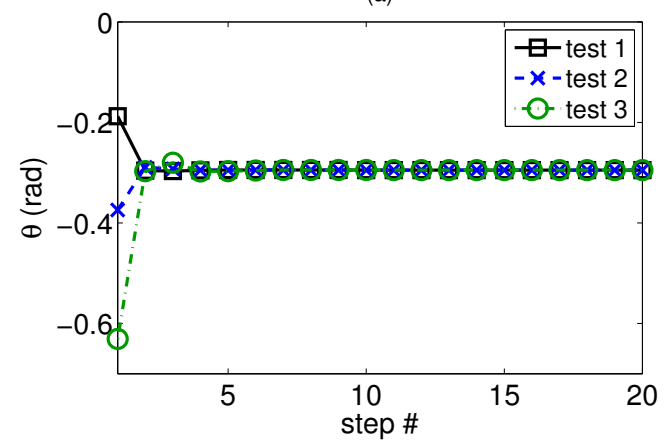

(b)

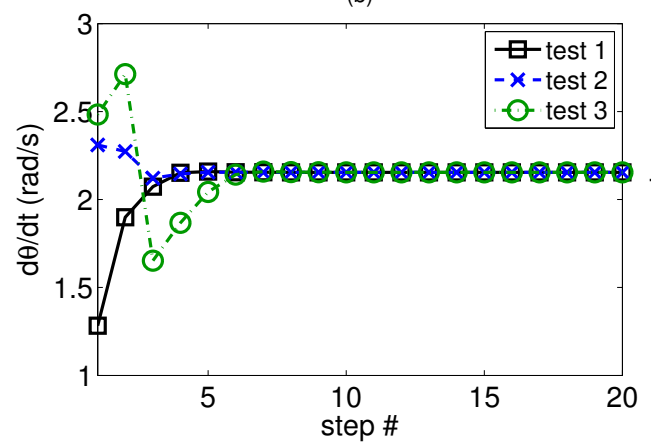

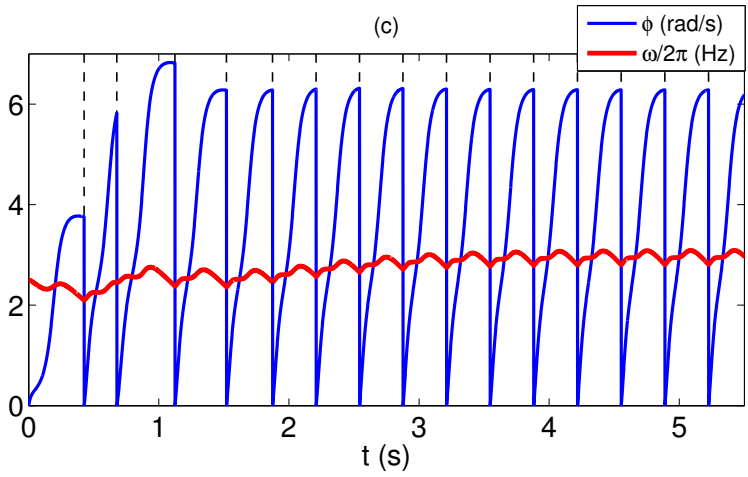

(d)

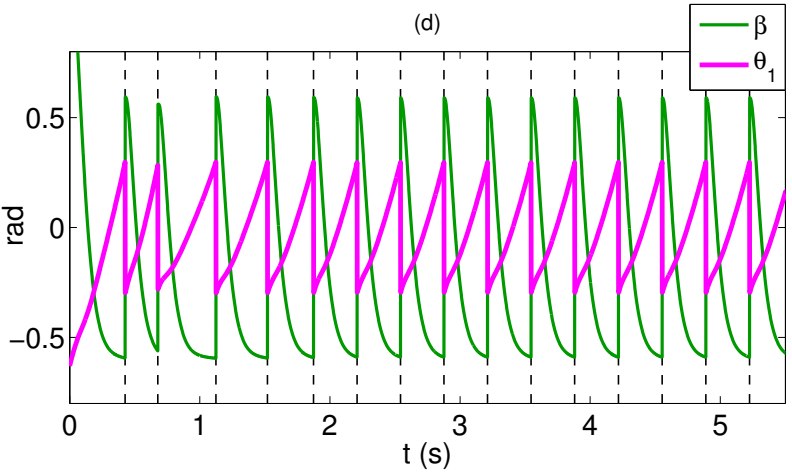

Figure 13: Dynamic walker with knees and finite hip mass to foot mass ratio: simulation with knees locked for different initial conditions $(\theta, \dot{\theta})$. Vertical dashed lines represent ground collisions. (a) Initial stance leg angle at each step. (b) Initial stance leg angular velocity at each step. (c) AFO phase and frequency. (d) Stance angle and inter-leg separation angle. Simulation parameters: coupling parameter $\epsilon=40$, learning constant $\eta=8$, final equilibrium angle $\beta_{e q, f}=-0.6 \mathrm{rad}$, stance leg torque gain $\hat{\tau}_{h, o}=20.4375 \mathrm{~N}$-m, virtual spring constant $\hat{\kappa}=40.875$ $\mathrm{N}-\mathrm{m} / \mathrm{rad}$, damping coefficient $\hat{\nu}=3.8218 \mathrm{~N}-\mathrm{m}-\mathrm{s} / \mathrm{rad}$.

and (2) the impact-like behavior of the knee joint when the swing leg is extended. For the present study the ratio of hip to foot mass chosen was 10.

The modified dynamic walker, shown in Figure 12, is based on the model presented in Fu [5]; it features curved feet and assumes a locking mechanism for the knees. The model's walking gait consists of two qualitatively distinct modes. The first mode begins at toe-off with the stance leg fully extended at its knee joint locked. The swing leg's knee joint is free to rotate, thereby allowing the foot to clear the ground. The second mode begins when the swing leg becomes fully extended, at which point the knee joint becomes locked; thus the swing leg strikes the ground as a single rigid body. Details on the dimensioning of the dynamic walker's variables and the walker's mode transitions are given in Appendix C.

In order to reduce the dimensionality of the model we removed the online Fourier analysis and employed an AFO that uses the inter-leg angle $\beta$ directly as its input:

$$
\begin{aligned}
& \dot{\phi}=\omega-\epsilon \beta(t) \sin \phi \\
& \dot{\omega}=-\epsilon \beta(t) \sin \phi
\end{aligned}
$$

Unlike the previous model this AFO completes one cycle of $\phi(0$ to $2 \pi)$ per single stride.

To isolate the effects of the finite mass ratio, we ran simulations from different initial conditions keeping the walker's knees locked. Initial conditions were picked from within the walker's basin of attraction (Figure 16); the initial angular velocities were made dimensional using the formulas in Appendix C. Simulations showed that, although the resulting slope of $\phi$ is considerably less uniform, the stabilizing effects of the AFO are still present. As shown on Figure 13, the walker was able to reach the fixed point after only a few steps. The fixed-point stepping 

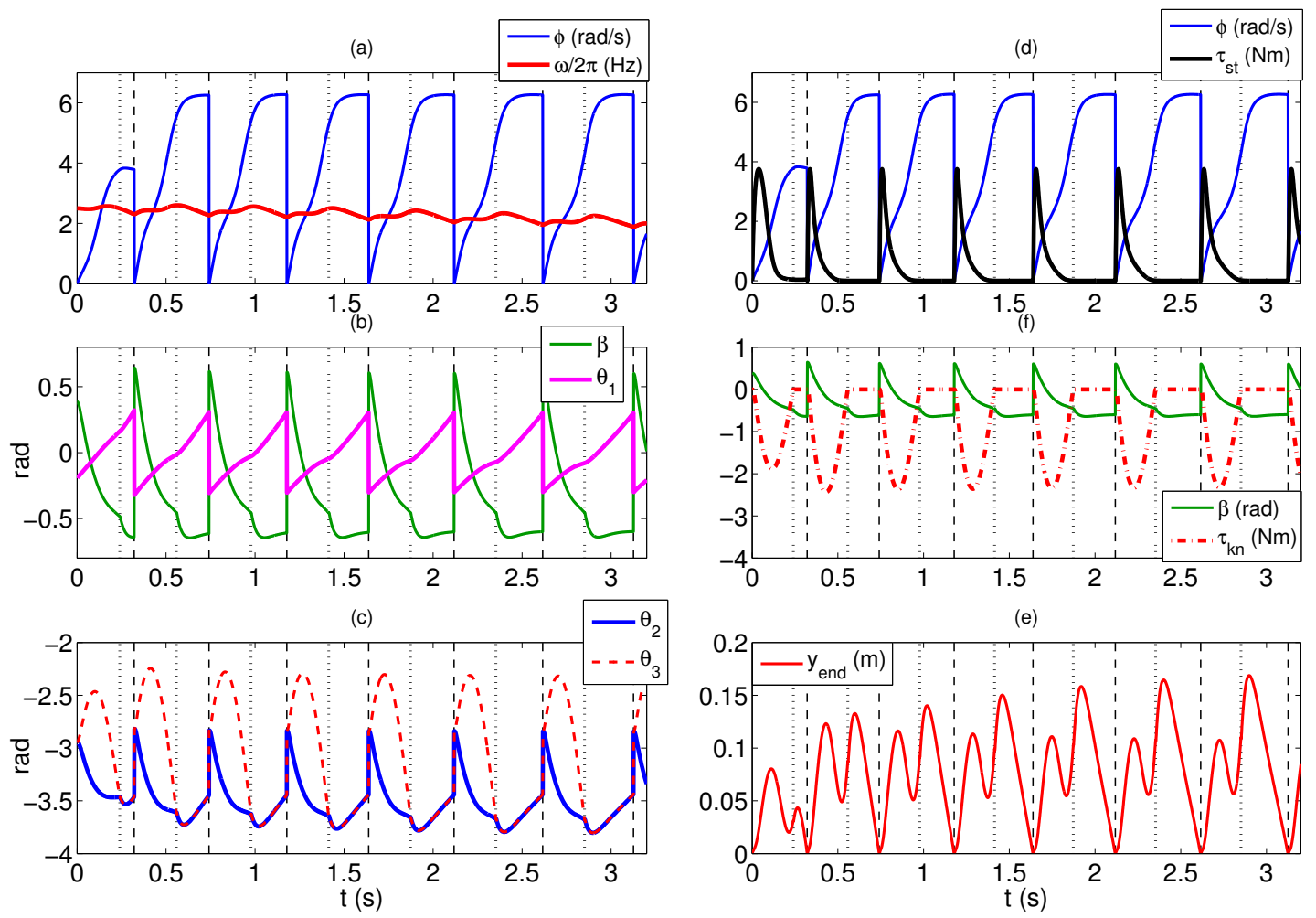

Figure 14: Dynamic walker with knees: simulation with ratio of hip spring constant to knee spring constant of 20. Vertical dotted lines represent knee collisions; vertical dashed lines represent ground collisions. (a) AFO phase and frequency. (b) Stance angle and inter-leg separation angle. (c) Hip joint and knee joint angles with respect to vertical. (d) Stance leg torque. (e) Knee joint torque. (f) Swing leg foot elevation above ground. Simulation parameters: coupling parameter $\epsilon=40$, learning constant $\eta=8$, final equilibrium angle $\beta_{\text {eq, } f}=-0.6 \mathrm{rad}$, stance leg torque gain $\hat{\tau}_{h, o}=20.4375 \mathrm{~N}-\mathrm{m}$, virtual spring constant $\hat{\kappa}=40.875 \mathrm{~N}-\mathrm{m} / \mathrm{rad}$, damping coefficient $\hat{\nu}=3.8218$ $\mathrm{N}-\mathrm{m}-\mathrm{s} / \mathrm{rad}$, knee joint virtual spring constant $\hat{\kappa}_{k n}=2.0438 \mathrm{~N}-\mathrm{m} / \mathrm{rad}$.

frequency was $2.98 \mathrm{~Hz}$, in close agreement with the value predicted by the original walker model, $3.11 \mathrm{~Hz}$ after dimensionalization. The mean forward speed of the walker was $1.730 \mathrm{~m} / \mathrm{s}$.

As a preamble to designing a control for the angular motion of the knee joint, we tested the effect of the impulsive forces at knee-lock on the walker's gait. We ran simulations in which the knee joint was allowed to rotate passively, constrained only by a virtual torsional spring. Although we currently lack a systematic procedure for determining the torsional spring constant, we found a stable gait by selecting a value $\hat{\kappa}_{k n}$ equivalent to $5 \%$ the spring constant of the hip joint $(\hat{\kappa})$. The selected value $\hat{\kappa}_{k n}$ also allowed for the swing leg's foot to clear the ground. The simulated gait is shown in Figure 14. However a significant change occurred in the stepping frequency, which decreased from $2.98 \mathrm{~Hz}$ in the simulation with rigid legs to $2.01 \mathrm{~Hz}$. Step length on its part remained essentially unchanged; thus the walker's forward speed decreased to $1.185 \mathrm{~m} / \mathrm{s}$.

Simulations at higher values of $\hat{\kappa}_{k n}$ did not yield stable gaits except by increasing the stance torque gain; otherwise the walker would fall down after a few steps. Figure 15 shows an example simulation in which $\hat{\kappa}_{k n}$ was increased to $10 \%$ the spring constant on the hip and the stance torque was increased by $60 \%$. In this case the stepping frequency increased back to $2.81 \mathrm{~Hz}$ and the forward speed to $1.696 \mathrm{~m} / \mathrm{s}$. Given that the walker is more sensitive to velocity than angle perturbations (Figures 8(a) and 8(b)), a probable explanation for the loss of stability is the velocity perturbations caused by the impulsive force occurring at knee lock. While a single velocity perturbation can be tolerated as long as it occurs within the basin of attraction, repeated perturbations can drive the walker outside the basin by making it cross the "passive walker" boundary (Figure 11).

Although velocity perturbations due to knee collision could be compensated with velocity feedback acting on the stance torque, such approach is not desirable. Our AFO-based control aims to stabilize the walker using the 

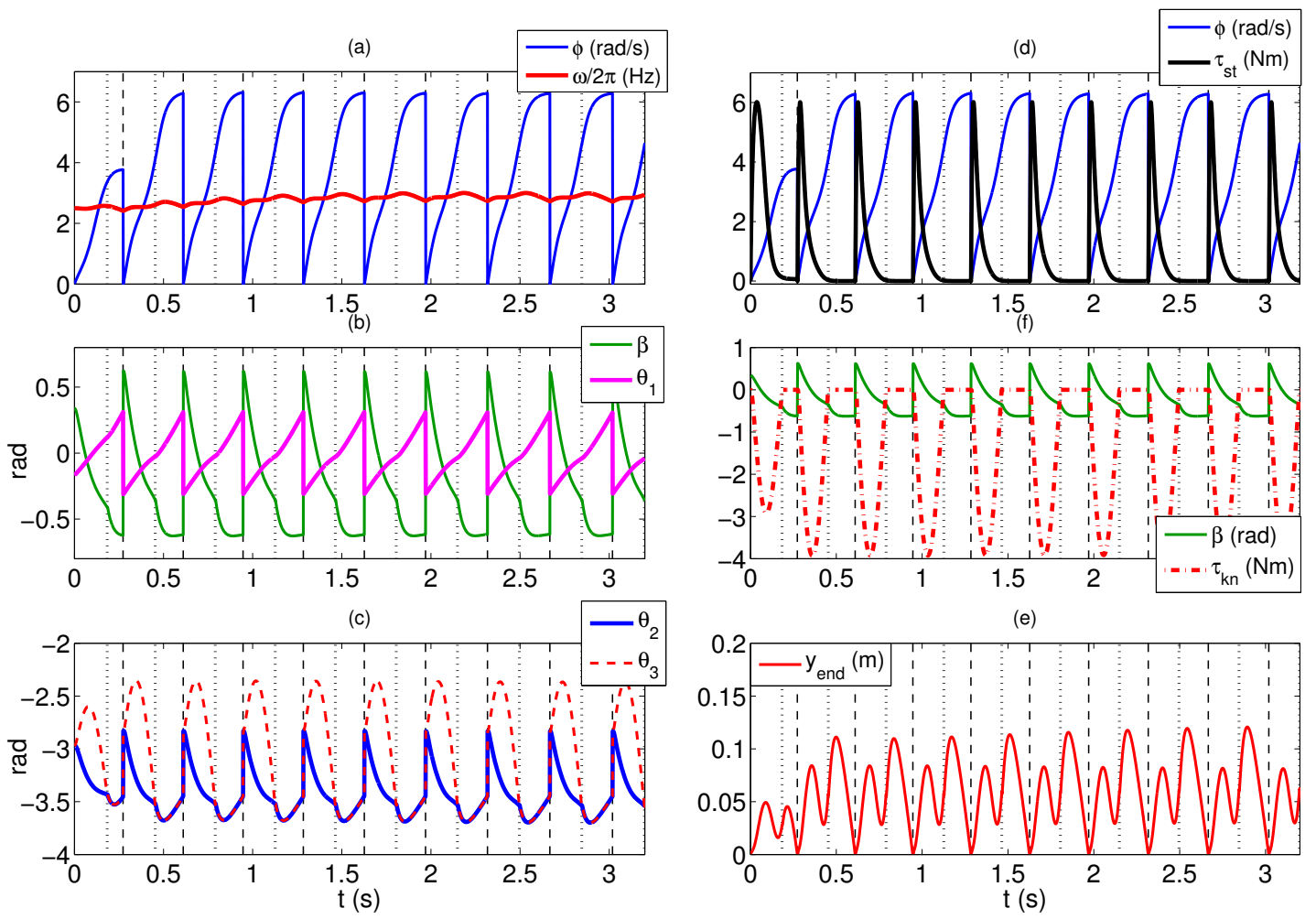

Figure 15: Dynamic walker with knees: simulation with ratio of hip spring constant to knee spring constant of 10. Vertical dotted lines represent knee collisions; vertical dashed lines represent ground collisions. (a) AFO phase and frequency. (b) Stance angle and inter-leg separation angle. (c) Hip joint and knee joint angles with respect to vertical. (d) Stance leg torque. (e) Knee joint torque. (f) Swing leg foot elevation above ground. Simulation parameters: coupling parameter $\epsilon=40$, learning constant $\eta=8$, final equilibrium angle $\beta_{\text {eq, } f}=-0.6 \mathrm{rad}$, stance leg torque gain $\hat{\tau}_{h, o}=32.7 \mathrm{~N}-\mathrm{m}$, virtual spring constant $\hat{\kappa}=40.875 \mathrm{~N}-\mathrm{m} / \mathrm{rad}$, damping coefficient $\hat{\nu}=3.8218 \mathrm{~N}-\mathrm{m}-\mathrm{s} / \mathrm{rad}$, knee joint virtual spring constant $\hat{\kappa}_{k n}=4.0875 \mathrm{~N}-\mathrm{m} / \mathrm{rad}$.

phase of an angular trajectory as its sole feedback. A better alternative is to design a torque profile for the knee joint similar to the spring-damper torque profile we employed on the hip joint of the swing leg (equation (14)). The damping component would in principle reduce the intensity of the impact at knee-lock. However, the equilibrium trajectory of the knee spring needs to be designed such that it minimizes velocity perturbations not only at knee lock but throughout the first mode of the walker's gait.

\section{Discussion}

We investigated the capabilities of a CPG-like control based on an adaptive frequency oscillator to generate globally stable gait cycles in a bipedal walking model. First we demonstrated how a feedback system formed by an AFO and inverted pendulum representing the stance leg and the lumped hip mass of the walker can generate orbitally stable limit cycles. The key finding was that the frequency of the limit cycle can be controlled independently of the swing amplitude by applying an arbitrary gain $\left(\tau_{h o}\right)$ to the torque acting on the pendulum. This contrasts with CPG control of bipedal walkers based on resonance tuning $[3,26]$ in which the oscillator entrains itself to the natural frequency of the swing leg.

The small ratio of leg mass to hip mass in the SWM allows decoupling the movement of the swing leg from that of the stance leg. The practical consequence of this property is the ability to control the stance angle $\theta^{*}$ and the angular velocity $\dot{\theta}^{*}$ of the fixed point independently (see Figure 7).

In spite of its greater complexity with respect to the inverted-pendulum control, the full walker control preserved 


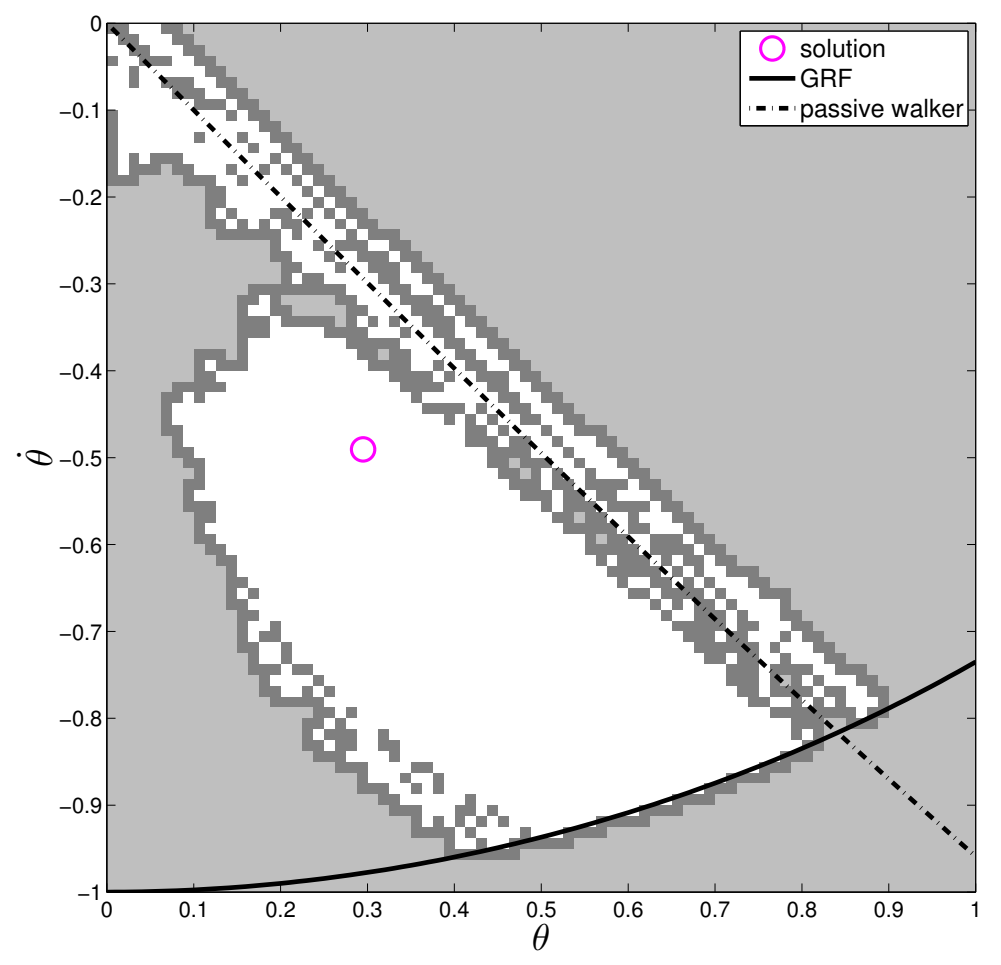

Figure 16: Basin of attraction using exact algorithm with normal behavior (period-1) of the walker. The gait solution is $\theta^{*}=0.29501$ and $\dot{\theta}^{*}=-0.49043$.

the basic relationship of proportionality between torque amplitude and step frequency. Thus we have demonstrated that CPG-based control of a bipedal walker does not necessarily have to tune itself to the resonant frequency of the walker's legs. Similarly to the model by Wisse [18], the virtual spring constant has the role of stabilizing the gait, but is not determinant of the step frequency. On the other hand, the energetic efficiency of the AFO-based control for different stride frequencies still needs to be studied.

Simulations for different values of $\epsilon$ suggested that this parameter may have an optimum value for walking control, beyond which the region of feasible gait solutions (Figure 7) begins to contract. However, the influence of $\epsilon$ and other AFO parameters needs to be studied in a more systematic fashion. The absence of $\epsilon$ in (5) suggests that this parameter does not have a significant role in determining steady-state values of step frequency and stride length. But at the same time, the coupling strength $\epsilon$ determines the rate of adaptation of AFO frequency [7], and a such may have a critical role in maintaining stability as the walker's gait progresses towards steady state. There is a need for a design methodology for AFO parameters (and CPG-based walking controls in general) to obtain arbitrary gait cycles in terms of frequency, amplitude or waveform [20].

To determine the global stability of a particular gait solution, we developed a fast algorithm that reduces the dimensionality of the walker's landmark state (15) by resetting the AFO at the end of every AFO cycle, which represents two steps of the walker. The algorithm has the advantage of speed because it uses recursion. The question is how much the behavior of the walker is altered by the resetting of $\phi$ and $\omega$. If the walker's gait is initially at the fixed point $\mathbf{z}^{*}$, resetting will not make any difference provided that $\omega^{+}$is exactly equal to $\omega^{*}$. For slightly different values of $\omega^{+}$, the walker will either produce a stable period-2 gait in the vicinity of $\mathbf{z}^{*}$, or fall down after executing a certain number of steps near $\mathbf{z}^{*}$. (A period-2 gait is a "limping" gait, i.e. one in which the stride of the right leg is different from that of the left leg. Thus the landmark state is repeated only every two strides.)

By way of comparison, we obtained the basin of attraction that results from the walker's normal behavior, i.e. walking without resetting the AFO. Although this algorithm generates an exact basin of attraction, it has the disadvantage of being considerably slower because it does not allow recursion. For an initial condition $b_{j}$ to be included in the basin, it has to generate the complete sequence of strides ending in $\Gamma\left(\mathbf{z}^{*}\right)$. If we assume the state 
space of the walker to be divided into $n_{c} \times n_{c}$ cells, the number of computations required by the exact algorithm is of order $O\left(n_{c}^{3}\right)$. By contrast, the fast algorithm with phase resetting is of order $O\left(n_{c}^{2}\right)$. The result of the exact algorithm for the same fixed point is shown in Figure 16. The basin from the exact algorithm is somewhat smaller because it avoids convergence to gaits in the vicinity of but different from $\mathbf{z}^{*}$.

With a view to implementing the AFO-based control on an actual bipedal robot, we simulated the action of the AFO on a bipedal model featuring a finite ratio of hip mass to foot mass and articulated knee joints. Simulations showed that the finite mass ratio does not adversely affect the stability of the system. However, when the knees were allowed to bend passively under the action of a virtual spring it proved difficult to find stable gait solutions. This was not altogether unexpected because the virtual spring by itself can enforce an angle constraint but not a velocity one. Thus our next goal is to design a phase-driven control for the knee joints that preserves the overall behavior of the simple walking model (section 6), namely by rejecting the velocity perturbations caused by rotation of the knee joint and the impact at knee-lock. This problem is similar to the one studied by Garofalo [27], in which a multiDOF walking robot is forced to track the behavior of a simple walking model, in that case a spring-loaded inverted pendulum (SLIP) walker. The approach proposed there is to project the torques computed for a low-priority task onto the null space of the Jacobian matrix transpose of a higher priority task. A similar method may be applied in our case to ensure that the torques leading the swing leg to its final position do not interfere with the higher priority task of controlling the angle and velocity of the stance leg.

\section{Conclusion and future work}

We have presented a simple control method that allows simultaneous frequency tuning and stabilization of a bipedal walker by means of an adaptive frequency oscillator. The main results from this study are as follows:

- An inverted-pendulum model provided a basic demonstration of the existence of orbitally stable limit cycles in the closed-loop system formed by the AFO and the walker.

- AFO-driven walking control is not constrained to resonance tuning. Stride frequency can be controlled independently of the natural pendulum frequency of the walker's legs by adjusting an independent control gain.

- Stability was accomplished by a constraint in the form of a spring and damper combination, with a traveling equilibrium point linked to the oscillator phase. This strategy preserved the ability to control stride frequency independently.

- To determine global stability of the AFO-driven walking control, we developed a recursive algorithm that allows fast generation of the basin of attraction of a fixed point through periodic resetting of the AFO. AFO resetting preserves the overall attractor properties of the fixed point by ensuring that the gait converges to a small vicinity of the fixed point, although it does not guarantee a sustained gait in that vicinity.

- Regarding the walking model with knees, the stabilizing action of the AFO is preserved for a finite mass ratio but is generally insufficient for knee joints rotating in a purely passive manner.

Future work will focus on developing a simple AFO-based control for the knee joints that specifically rejects velocity disturbances. In order to achieve coordination among limb segments, the control can be expanded to include multiple coupled oscillators. By introducing a certain level of independence among leg actuators, it should also provide provide better disturbance rejection capabilities. We will also investigate the use of AFO-driven control for generating stable running gaits. In order to model running, the bipedal model will have to be enhanced with biologically-inspired properties such as compliance and foot geometry. 
Initialize basin of attraction: $A_{c} \leftarrow \Gamma\left(\mathbf{z}^{*}\right)$;

Initialize set of visited cells: $V_{c} \leftarrow A_{c}$;

repeat

$A_{c, \text { old }} \leftarrow A_{c}$;

Generate boundary: $B_{c} \leftarrow\left(\right.$ dilation $\left.\left(A_{c}\right)-V_{c}\right)$ see Figure 10(a);

Label cells of $B_{c}$ as $b_{j}\left(j=1 \ldots \operatorname{numel}\left(B_{c}\right)\right)$;

while $\operatorname{numel}\left(B_{c}\right)>0$ do

for $b_{j}$ with lowest index $j$ do

$\mathbf{z} \leftarrow \Gamma^{-1}\left(b_{j}\right)$ walker state associated with $b_{j} ;$

$Z \leftarrow\{\mathbf{z}\}$ initialize set of walker states

repeat

$\mathbf{z} \leftarrow \mathbf{f}^{2}(\mathbf{z})$ attempt two strides $;$

if $\mathbf{z}$ is valid then $Z \leftarrow Z \cup\{\mathbf{z}\}$;

Reset $(\phi, \omega)$;

until z not valid or $\left(\Gamma(Z) \cap V_{c} \neq \varnothing\right)$ see Figure $10(b)$;

$V_{c} \leftarrow V_{c} \cup \Gamma(Z)$;

if $\Gamma(Z) \cap A_{c} \neq \varnothing$ then $A_{c} \leftarrow A_{c} \cup \Gamma(Z)$ see Figure $10(c)$;

end

Remove $b_{j}$ from $B_{c}$;

end

until numel $\left(A_{c}-A_{c, \text { old }}\right)=0$;

Table 1: Generation of the discretized basin of attraction: fast algorithm with resetting of AFO phase and frequency. (Note: the function numel() returns the number of elements in a set.) 


\section{References}

[1] C. Pinto and M. Golubitsky, "Central pattern generators for bipedal locomotion," J Math Biol, vol. 53, pp. 474-489, 2006.

[2] Y. Nakamura, T. Mori, M. Sato, and S. Ishii, "Reinforcement learning for a biped robot based on a cpg-actorcritic method," Neural Networks, vol. 20, pp. 723-735, 2007.

[3] B. W. Verdaasdonk, H. F. J. M. Koopman, and F. C. T. van der Helm, "Energy efficient walking with central pattern generators: from passive dynamic walking to biologically inspired control," Biological Cybernetics, vol. 101, pp. 49-61, 2009.

[4] J. Nakanishi, J. Morimoto, G. Endo, G. Cheng, S. Schaal, and M. Kawato, "Learning from demonstration and adaptation of biped locomotion,” Robotics and Autonomous Systems, vol. 47, pp. 79-91, 2004.

[5] C. Fu, F. Tan, and K. Chen, "A simple walking strategy for biped walking based on an intermittent sinusoidal oscillator," Robotica, vol. 28, pp. 869-884, 2010.

[6] L. Righetti and A. Ijspeert, "Programmable central pattern generators: an application to biped locomotion control," Proceedings IEEE International Conference on Robotics and Automation (ICRA), pp. 1585-1590, 2006.

[7] L. Righetti, J. Buchli, and A. Ijspeert, "Dynamic Hebbian learning in adaptive frequency oscillators," Physica $D$, vol. 216, no. 2, pp. 269-281, 2006.

[8] A. Ijspeert, J. Nakanishi, and S. Schaal, "Trajectory formation for imitation with nonlinear dynamical systems," Proceedings IEEE/RSJ International Conference on Intelligent Robots and Systems (IROS), 2001, vol. 2, pp. 752-757, 2001.

[9] S. Degallier, L. Righetti, S. Gay, and A. Ijspeert, "Toward simple control for complex, autonomous robotic applications: combining discrete and rhythmic motor primitives," Autonomous Robots, vol. 31, no. 2-3, pp. $155-181,2011$.

[10] A. Gams, A. Ijspeert, S. Schaal, and J. Lenarcic, "On-line learning and modulation of periodic movements with nonlinear dynamical systems," Autonomous Robots, vol. 27, no. 1, pp. 3-23, 2009.

[11] T. Petric, A. Gams, A. Ijspeert, and L. Zlajpah, "On-line frequency adaptation and movement imitation for rhythmic robotic tasks," International Journal of Robotics Research, vol. 30, no. 14, pp. 1775-1788, 2011.

[12] D. Hobbelen and M. Wisse, Limit cycle walking (in "Humanoid Robots, Human-Like Machines"), M. Hackel, Ed. Vienna, Austria: I-Tech Education and Publishing, 2007.

[13] A. D. Kuo, "Energetics of actively powered locomotion using the simplest walking model," Journal of Biomechanical Engineering, vol. 124, pp. 113-120, 2002.

[14] A. Kuo, J. Donelan, and A. Ruina, "Energetic consequences of walking like an inverted pendulum: Step-to-step transitions," Exercise and Sport Sciences Reviews, vol. 33, no. 2, 2005.

[15] M. Vukobratovic and A. Rodic, "Contribution to the integrated control of biped locomotion mechanisms," International Journal on Humanoid Robotics, vol. 4, no. 1, pp. 49-95, 2007.

[16] M. Garcia, A. Chatterjee, A. Ruina, and M. Coleman, "The simplest walking model: Stability, complexity and scaling,” ASME Journal of Biomechanical Engineering, vol. 120, pp. 281-288, 1998.

[17] A. Schwab and M. Wisse, "Basin of attraction of the simplest walking model," Proceedings of DETCO1 / ASME 2001 Design Engineering Technical Conferences and Computers and Information in Engineering Conference, 2001. 
[18] M. Wisse, A. Schwab, R. van der Linde, and F. van der Helm, "How to keep from falling forward: elementary swing leg action for passive dynamic walkers," Robotics, IEEE Transactions on, vol. 21, no. 3, pp. 393 - 401, june 2005 .

[19] D. Hobbelen and M. Wisse, "Swing-leg retraction for limit cycle walkers improves disturbance rejection," Robotics, IEEE Transactions on, vol. 24, no. 2, pp. 377 -389, april 2008.

[20] A. Ijspeert, "Central pattern generators for locomotion control in animals and robots: a review," Neural Networks, vol. 21, pp. 642-653, 2008.

[21] J. Buchli and A. Ijspeert, "Self-organized adaptive legged locomotion in a compliant quadruped robot," $A u$ tonomous Robots, vol. 25, no. 4, pp. 331-347, 2008.

[22] I. Nagrath and M. Gopal, Control Systems Engineering, 5th ed. UK: Anshan Ltd., 2008.

[23] R. Neptune, K. Sasaki, and S. Kautz, "The effect of walking speed on muscle function and mechanical energetics," Gait \& Posture, vol. 28, no. 1, pp. 135-143, 2008.

[24] J. Xu, R. Guttalu, and C. Hsu, "Domains of attraction for multiple limit cycles of coupled Van der Pol equations by simple cell mapping," International Journal of Non-Linear Mechanics, vol. 20, no. 5-6, pp. 507-517, 1985.

[25] R. Jain, R. Kasturi, and B. Schunck, Machine Vision, 1st ed. McGraw-Hill Interntional Editions, 1995.

[26] R. Ronsse, J. van den Kieboom, and A. J. Ijspeert, "Automatic resonance tuning and feedforward learning of biped walking using adaptive oscillators," Proceeding of the Conference in Multibody Dynamics 2011, ECCOMAS Thematic Conference, 2011.

[27] G. Garofalo, C. Ott, and A. Albu-Schaffer, "Walking control of fully actuated robots based on the bipedal slip model," IEEE International Conference on Robotics and Automation (ICRA), pp. 1456-1463, 2012.

\section{A Inverted-pendulum system with backlash}

The closed-loop system of Figure 2 is described in frequency domain by

$$
\theta+P(j \omega) W(j \omega) N(\Theta) \theta-P(j \omega) \tau_{h o} u=0
$$

The dynamics of the inverted pendulum (Figure 1(b)), after linearization and normalization, are given by the transfer function $P(s)=1 /\left(s^{2}-1\right)$. The lateral "walls" are modeled as a combination of a spring of stiffness $k$ and damper with coefficient $c$ to yield $W(s)=c s+k$. In order to find a limit-cycle solution $(\Theta, \omega)$ for $(28)$, in addition to the backlash describing function $N(\Theta)$ we need a describing function for the AFO. Using the low-pass assumption (section 3), any signal in the system of Figure 1(b) can be approximated by a sinusoid. Thus given the AFO output $u=\cos \phi$, the pendulum angular velocity $\Omega(t)$ can be expressed as a change in the amplitude and phase of $u(t)$ :

$$
\Omega=|\Omega| \cos (\phi+\gamma)
$$

Substituting $\Omega$ in (26) we obtain

$$
\dot{\phi}=\omega-\epsilon|\Omega| \cos (\phi+\gamma) \sin \phi
$$

The rate of convergence of the AFO to a limit cycle is faster than the rate of adaptation of the frequency parameter $\omega$ [7]. Therefore, the mean effective frequency $\bar{\omega}$ of the limit cycle [21] can be approximated as

$$
\bar{\omega}=\omega+\frac{1}{2} \epsilon|\Omega| \sin \gamma
$$

Assuming that the system has a stable limit cycle $(\Theta, \omega)$, the frequency adaptation of the AFO ends when $\bar{\omega}=\omega$. From (31) this yields the condition $\gamma=0$. Applying this result to (29) we obtain $\Omega=|\Omega| \cos \phi=|\Omega| u$. But given that $\Omega=j \omega \theta$, the AFO output for the limit cycle in frequency domain is simply 


$$
u(j \omega)=\frac{j \omega \theta(j \omega)}{\omega \Theta}=\frac{j}{\Theta} \theta(j \omega)
$$

Substituting $u$ in (28) yields

$$
1+P(j \omega) W(j \omega) N(\Theta)-j\left(\tau_{h o} / \Theta\right) P(j \omega)=0
$$

which can be rewritten as $N(\Theta)-Q(\Theta, \omega)=0$ where

$$
Q(\Theta, \omega)=\frac{j\left(\tau_{h o} / \Theta\right) P(j \omega)-1}{P(j \omega) W(j \omega)}
$$

$Q(\Theta, \omega)$ is a complex function of which the real and the imaginary part are, respectively,

$$
\begin{aligned}
& \operatorname{Re}\{Q(\Theta, \omega)\}=\frac{k\left(\omega^{2}+1\right)+\left(\tau_{h o} / \Theta\right) c \omega}{c^{2} \omega^{2}+k^{2}} \\
& \operatorname{Im}\{Q(\Theta, \omega)\}=-\frac{c \omega\left(\omega^{2}+1\right)-\left(\tau_{h o} / \Theta\right) k}{c^{2} \omega^{2}+k^{2}}
\end{aligned}
$$

A solution $(\Theta, \omega)$ represents a limit cycle of the inverted pendulum. The orbital stability of the limit cycle can be determined by computing the gradients $\operatorname{Re}\left\{\frac{\partial Q}{\partial \omega}\right\}$ and $\operatorname{Im}\left\{\frac{\partial Q}{\partial \omega}\right\}$. A locally-stable limit cycle is one for which both gradients are negative.

\section{B Finding gait solutions for the bipedal walker using a linearized model}

A periodic gait cycle occurs when the initial conditions of one step are repeated at the beginning of the next step. We express a gait cycle solution as a set of values $\left(\Theta, \Omega, T_{s}\right)$ such that the initial conditions are $\theta(0)=\Theta$ and $\dot{\theta}(0)=\Omega$, and $T_{s}$ represents the period of one step. We will obtain approximate gait cycle solutions for the walker by linearizing the equations of motion about $\left[\begin{array}{cccc}\theta & \dot{\theta} & \beta & \dot{\beta}\end{array}\right]^{T}=\mathbf{0}$ and using simplified expressions for the hip torques. The stance leg torque of (13) is approximated by

$$
\tau_{h, s t}=\bar{\tau}_{h o} t e^{-\sigma t}
$$

where $\bar{\tau}_{h o}=2 \pi \tau_{h o} / T_{s}$ and $\sigma=2 \pi /\left(K_{s t} T_{s}\right)$. We still use (14) for the swing leg torque

$$
\tau_{h, s w}=k\left(\beta-\beta_{e q}\right)+c \dot{\beta}
$$

but prescribe $\beta_{e q}$ to be constant. This approximation is acceptable under the assumption that the swing leg does not perturb the motion of the stance leg, as long as the final inter-leg aperture angle $\beta$ matches that of the nonlinear system (1)(2). Defining $\psi=\beta-\beta_{e q}$ and substituting $\tau_{h, s t}$ and $\tau_{h, s w}$, the small-angle approximation of (2) yields

$$
\ddot{\psi}+c \dot{\psi}+\left(\cos \beta_{e q}+k\right) \psi=\theta-\bar{\tau}_{h o} t e^{-\sigma t}-\sin \beta_{e q}
$$

We define $\gamma \equiv-\sin \beta_{e q}, \omega_{n}^{2} \equiv \cos \beta_{e q}+k$ and select $c=2 \omega_{n}$ to make the swing leg critically damped. Substituting these values into (39) and performing small-angle approximation on (1) allows us to write the following set of linearized equations of motion:

$$
\begin{aligned}
\ddot{\theta}-\theta & =-\bar{\tau}_{h o} t e^{-\sigma t} \\
\ddot{\psi}+2 \omega_{n} \dot{\psi}+\omega_{n}^{2} \psi & =\theta-\bar{\tau}_{h o} t e^{-\sigma t}+\gamma
\end{aligned}
$$

The task is to find a periodic gait solution for (40) and (41) subject to initial conditions

$$
\begin{aligned}
\theta(0) & =\Theta \\
\dot{\theta}(0) & =-\Omega \\
\psi(0) & =2 \Theta-\beta_{e q} \\
\dot{\psi}(0) & =0
\end{aligned}
$$


with jump conditions

$$
\begin{aligned}
\theta^{+} & =-\theta^{-} \\
\dot{\theta}^{+} & =\cos 2 \theta^{-} \dot{\theta}^{-} \\
\psi^{+} & =-2 \theta^{-}-\beta_{e q} \\
\dot{\psi}^{+} & =\cos 2 \theta^{-}\left(1-\cos 2 \theta^{-}\right) \dot{\theta}^{-}
\end{aligned}
$$

The fixed point of the gait cycle is enforced by the boundary conditions

$$
\begin{aligned}
& \theta^{+}\left(T_{s}\right)=\Theta \\
& \dot{\theta}^{+}\left(T_{s}\right)=-\Omega \\
& \psi^{+}\left(T_{s}\right)=2 \Theta-\beta_{e q}
\end{aligned}
$$

We solve for $\theta$ by first proposing the nonhomogeneous solution

$$
\theta_{n h}=B_{1} e^{-\sigma t}+B_{2} t e^{-\sigma t}
$$

Applying $\theta_{n h}$ to (40),

$$
\left(B_{1} \sigma^{2}-2 B_{2} \sigma-B_{1}\right) e^{-\sigma t}+\left(B_{2} \sigma^{2}-B_{2}\right) t e^{-\sigma t}=-\bar{\tau}_{h o} t e^{-\sigma t}
$$

which yields the coefficients

$$
B_{1}=-\frac{2 \sigma \bar{\tau}_{h o}}{\left(\sigma^{2}-1\right)^{2}}, B_{2}=-\frac{\bar{\tau}_{h o}}{\sigma^{2}-1}
$$

The complete solution for $\theta$ is given by

$$
\begin{aligned}
& \theta=A_{1} e^{t}+A_{2} e^{-t}+\left(B_{1}+B_{2} t\right) e^{-\sigma t} \\
& \dot{\theta}=A_{1} e^{t}-A_{2} e^{-t}+\left(-B_{1} \sigma+B_{2}-B_{2} \sigma t\right) e^{-\sigma t}
\end{aligned}
$$

where initial conditions (42) and (43) applied respectively to (56) and (57) yield the coefficients

$$
\begin{aligned}
& A_{1}=\frac{1}{2}\left(\Theta-\Omega+B_{1}(\sigma-1)-B_{2}\right) \\
& A_{2}=\frac{1}{2}\left(\Theta+\Omega-B_{1}(\sigma+1)+B_{2}\right)
\end{aligned}
$$

We proceed now to solve for $\psi$. Substituting the solution for $\theta$ (56) into (41) yields

$$
\ddot{\psi}+2 \omega_{n} \dot{\psi}+\omega_{n}^{2} \psi=A_{1} e^{t}+A_{2} e^{-t}+B_{1} e^{-\sigma t}+B_{2} t e^{-\sigma t}-\bar{\tau}_{h o} t e^{-\sigma t}+\gamma
$$

Therefore we propose the nonhomogeneous solution

$$
\psi_{n h}=E_{1} e^{t}+E_{2} e^{-t}+F_{1} e^{-\sigma t}+F_{2} t e^{-\sigma t}+F_{3}
$$

Applying $\psi_{n h}$ to (41),

$$
\begin{array}{r}
E_{1} e^{t}+E_{2} e^{-t}+\left(F_{1} \sigma^{2}-F_{2} \sigma\right) e^{-\sigma t}-F_{2} \sigma e^{-\sigma t}+F_{2} \sigma^{2} t e^{-\sigma t} \\
+2 \omega_{n} E_{1} e^{t}-2 \omega_{n} E_{2} e^{-t}+2 \omega_{n}\left(-F_{1} \sigma+F_{2}\right) e^{-\sigma t}-2 \omega_{n} F_{2} \sigma t e^{-\sigma t} \\
+\omega_{n}^{2} E_{1} e^{t}+\omega_{n}^{2} E_{2} e^{-t}+\omega_{n}^{2} F_{1} e^{-\sigma t}+\omega_{n}^{2} F_{2} t e^{-\sigma t}+\omega_{n}^{2} F_{3} \\
=A_{1} e^{t}+A_{2} e^{-t}+B_{1} e^{-\sigma t}+B_{2} t e^{-\sigma t}-\bar{\tau}_{h o} t e^{-\sigma t}+\gamma
\end{array}
$$


which yields the coefficients

$$
\begin{aligned}
E_{1} & =\frac{A_{1}}{1+2 \omega_{n}+\omega_{n}^{2}} \\
E_{2} & =\frac{A_{2}}{1-2 \omega_{n}+\omega_{n}^{2}} \\
F_{1} & =\frac{B_{1}}{\sigma^{2}-2 \omega_{n} \sigma+\omega_{n}^{2}}-2 \frac{\left(\omega_{n}-\sigma\right)\left(B_{2}-\bar{\tau}_{h o}\right)}{\left(\sigma^{2}-2 \omega_{n} \sigma+\omega_{n}^{2}\right)^{2}} \\
F_{2} & =\frac{B_{2}-\bar{\tau}_{h o}}{\sigma^{2}-2 \omega_{n} \sigma+\omega_{n}^{2}} \\
F_{3} & =\frac{\gamma}{\omega_{n}^{2}}
\end{aligned}
$$

The complete solution for $\psi$ adds an homogeneous term to $\psi_{n h}$ to yield

$$
\begin{aligned}
& \psi=\left(D_{1}+D_{2} t\right) e^{-\omega_{n} t}+E_{1} e^{t}+E_{2} e^{-t}+\left(F_{1}+F_{2} t\right) e^{-\sigma t}+F_{3} \\
& \dot{\psi}=\left(-D_{1} \omega_{n}+D_{2}-D_{2} \omega_{n} t\right) e^{-\omega_{n} t}+E_{1} e^{t}-E_{2} e^{-t}+\left(-F_{1} \sigma+F_{2}-F_{2} \sigma t\right) e^{-\sigma t}
\end{aligned}
$$

where initial conditions (44) and (45) applied respectively to (64) and (65) yield the coefficients

$$
\begin{aligned}
& D_{1}=2 \Theta-\beta_{e q}-E_{1}-E_{2}-F_{1}-F_{2} \\
& D_{2}=D_{1} \omega_{n}-E_{1}+E_{2}+F_{1} \sigma-F_{2}
\end{aligned}
$$

The solutions for $\theta$ (56), $\dot{\theta}(57), \psi(64)$ and $\dot{\psi}(65)$ are functions of the gait parameters $\Theta, \Omega$ and $T_{s}$. In order to solve for these, we need to use the jump conditions and boundary conditions of the periodic gait. Boundary condition (50) applied to the jump condition (46) yields

$$
\theta^{-}\left(T_{s}\right)=-\theta^{+}\left(T_{s}\right)=-\Theta
$$

Substituting $\theta^{-}\left(T_{s}\right)$ in (56) yields

$$
-\Theta=A_{1} e^{T_{s}}+A_{2} e^{-T_{s}}+\left(B_{1}+B_{2} T_{s}\right) e^{-\sigma T_{s}}
$$

Boundary condition (51) applied to the jump condition (47) yields

$$
-\Omega=\cos 2 \Theta \dot{\theta}^{-}\left(T_{s}\right)
$$

Substituting $\dot{\theta}^{-}\left(T_{s}\right)$ in (57) yields

$$
-\frac{\Omega}{\cos 2 \Theta}=A_{1} e^{T_{s}}-A_{2} e^{-T_{s}}+\left(-B_{1} \sigma+B_{2}-B_{2} \sigma T_{s}\right) e^{-\sigma T_{s}}
$$

Finally, applying the boundary condition (52) to the jump condition (48) yields

$$
\psi^{-}\left(T_{s}\right)=-2 \Theta-\beta_{e q}
$$

Substituting $\psi^{-}\left(T_{s}\right)$ in (64) yields

$$
-2 \Theta-\beta_{e q}=\left(D_{1}+D_{2} T_{s}\right) e^{-\omega_{n} T_{s}}+E_{1} e^{T_{s}}+E_{2} e^{-T_{s}}+\left(F_{1}+F_{2} T_{s}\right) e^{-\sigma T_{s}}+F_{3}
$$

Thus the gait parameters $\Theta, \Omega$ and $T_{s}$ are the solution to (68), (70) and (72). 


\section{Dynamic walking model with knee joints and finite mass ratio}

The dynamic walker with knees (Figure 12) features a "thigh" segment with a mass $M=0.4167 \mathrm{~kg}$ centered at the hip joint and a "shank" segment with a mass $m=0.0833 \mathrm{~kg}$ located at the distal point. The centroidal moments of inertia are $I_{t}=0.005 \mathrm{~kg}-\mathrm{m}^{2}$ for the thigh and $I_{s}=0.001 \mathrm{~kg} \mathrm{~m}^{2}$ for the shank. The total length of the leg is $l=1 \mathrm{~m}$ and the feet have radii of $0.2 \mathrm{~m}$.

The state and control variables of the original walking model are defined in dimensionless space. In order to be used on the dynamic walker with knees, these variables are made dimensional per the walker's physical parameters. To this end we will use the following values corresponding to the leg's knee-locked configuration:

- Center of mass: $c_{1}=\frac{M}{m+M} l$

- Moment of inertia about the distal point $\left[\begin{array}{ll}o_{x} & o_{y}\end{array}\right]^{T}: I_{o}=I_{s}+I_{t}+M l^{2}$

- Moment of inertia about the hip joint : $I_{o}=I_{s}+I_{t}+m l^{2}$

Thus the dimensional variables of the walker with knees (denoted by the symbol ^) are obtained as follows:

- Angular velocity: $\dot{\hat{\theta}}=\sqrt{\frac{M g c_{1}}{I_{o}}} \dot{\theta}$

- Stance leg torque: $\hat{\tau}_{h, o}=2 M g c_{1} \tau_{h, o}$

- Swing leg spring constant: $\hat{\kappa}=m g l \kappa$

- Swing leg damping coefficient: $\hat{\nu}=2 \sqrt{\hat{\kappa} I_{h}}$

The mode transitions are based on the walker model by $\mathrm{Fu}$ [5]. The first transition occurs when the swing leg becomes fully extended and the knee joint becomes locked. The geometric condition for the transition is

$$
\theta_{2}-\theta_{3}=0
$$

Given the walker's state in generalized coordinates, $\mathbf{q}=\left[\begin{array}{lll}\theta_{1} & \theta_{2} & \theta_{3}\end{array}\right]^{T}$, and the inertia matrix $D(\mathbf{q})$, to find the joints' angular velocities $\dot{\mathbf{q}}^{+}$after knee lock we apply conservation of momentum as

$$
D\left(\mathbf{q}^{+}\right) \dot{\mathbf{q}}^{+}-D\left(\mathbf{q}^{-}\right) \dot{\mathbf{q}}^{-}=\left[\begin{array}{c}
0 \\
-P_{k} \\
P_{k}
\end{array}\right]
$$

where $P_{k}$ in the impulse occurring on the knee joint as a result of the locking action. The associated constraint is that the angular velocities of the thigh and the shank become equal after knee lock:

$$
\left[\begin{array}{lll}
0 & 1 & -1
\end{array}\right] \dot{\mathbf{q}}^{+}=0
$$

From (74) and (75) we solve for $\dot{\mathbf{q}}^{+}$and $P_{k}$.

The second transition occurs when the leading foot makes contact with the ground and the trailing leg becomes the swing leg. To derive the transition equations we define an extended state vector that includes the Cartesian coordinates of the lowest point on the trailing foot (Figure 12(b)):

$$
\mathbf{p}=\left[\begin{array}{llll}
o_{x} & o_{y} & \theta_{1} & \theta_{2}
\end{array}\right]^{T}
$$

The geometric conditions for the ground collision are

$$
\theta_{1}+\theta_{2}-\pi=0
$$

and

$$
o_{x}=o_{y}=0
$$


Given the inertia matrix $D_{p}(\mathbf{p})$, conservation of momentum yields

$$
D_{p}\left(\mathbf{p}^{+}\right) \dot{\mathbf{p}}^{+}-D_{p}\left(\mathbf{p}^{-}\right) \dot{\mathbf{p}}^{-}=J_{p}^{T}\left(\mathbf{p}^{-}\right)\left[\begin{array}{c}
P_{g, x} \\
P_{g, y}
\end{array}\right]
$$

where $\left[P_{g, x}, P_{g, y}\right]^{T}$ is the impulse (in Cartesian coordinates) on the leading foot at ground strike and $J_{p}^{T}(\mathbf{p})$ is the Jacobian matrix relating the velocities $\dot{\mathbf{p}}$ to the Cartesian velocity of the leading foot (point $\left[\begin{array}{ll}g_{x} & g_{y}\end{array}\right]^{T}$ in Figure 12(b)). The associated constraint is that the Cartesian velocity of the leading foot is zero after ground strike:

$$
J_{p}^{T}\left(\mathbf{p}^{+}\right) \dot{\mathbf{p}}^{+}=0
$$

Equations (79) and (80) yield the solutions for for $\dot{\mathbf{p}}^{+}$and $\left[P_{g, x}, P_{g, y}\right]^{T}$. 